\title{
What works in family planning interventions: A systematic review of the evidence
}

\author{
Lisa Mwaikambo, MPH${ }^{1}$, Ilene S. Speizer, $\mathrm{PhD}^{2}$, Anna Schurmann, $\mathrm{MPH}^{3}$, Gwen Morgan, \\ $\mathrm{PhD}^{4}$, and Fariyal Fikree, MD, $\mathrm{DrPH}^{5}$ \\ ${ }^{1}$ Center for Communication Programs, Johns Hopkins University Bloomberg School of Public \\ Health \\ ${ }^{2}$ Carolina Population Center and Department of Maternal and Child Health, University of North \\ Carolina at Chapel Hill Gillings School of Global Public Health \\ ${ }^{3}$ Sukshema Project, Karnataka Health Promotion Trust/Intrahealth \\ ${ }^{4}$ African Population and Health Research Center, Nairobi, Kenya \\ ${ }^{5}$ Independent Consultant, Global Health, Washington DC
}

\begin{abstract}
This study presents findings from a systematic review of evaluations of family planning interventions published between 1995 and 2008. Studies that used an experimental or quasiexperimental design or had another way to attribute program exposure to observed changes in fertility or family planning outcomes at the individual or population levels were included and ranked by strength of evidence. A total of 63 studies were found that met the inclusion criteria. The findings from this review are summarized in tabular format by the type of intervention (classified as supply-side or demand-side). About two-thirds of the studies found were on demand generation type-programs. Findings from all programs revealed significant improvements in knowledge, attitudes, discussion, and intentions. Program impacts on contraceptive use and use of family planning services were less consistently found and less than half of the studies that measured fertility or pregnancy-related outcomes found an impact. Based on the review findings, we identify promising programmatic approaches and propose directions for future evaluation research of family planning interventions.
\end{abstract}

\section{BACKGROUND}

By the early 1970s, international efforts to reduce rapid population growth in the developing world were well advanced. The vast majority of countries adopted voluntary family planning programs, which in most cases were part of their maternal and child health or primary health care systems (Sinding 2007). A golden era of family planning from 1970 to 1990-during which a reproductive revolution occurred in every region of the world except sub-Saharan Africa—was underway (Donaldson 1990; Donaldson and Tsui 1990).

\footnotetext{
Corresponding Author: Ilene S. Speizer, Department of Maternal and Child Health, Gillings School of Global Public Health, University of North Carolina at Chapel Hill, 206 W. Franklin St., CB 8120, Chapel Hill, NC 27516, Tel: 919-966-7411, Fax: 919-966-2391, ilene_speizer@unc.edu.

A previous version of this paper was presented in October, 2009 at the International Conference on Urban Health in Nairobi, Kenya and in November, 2009 at the International Conference on Family Planning: Research and Best Practices in Kampala, Uganda. At the time this work was undertaken, LM, AS, and FF were part of the MLE project team.
} 
Between the mid-1960s and the mid-1990s, average fertility in the developing world, including China, fell from around six children per woman over her reproductive lifetime to around three, a 50 percent decline. During the same period, the prevalence of contraceptive use among women increased from less than 10 percent to nearly 60 percent, but the rise was uneven (Sinding 2007).

Despite decades of research on the subject, considerable uncertainty exists about the processes and factors that motivate couples to limit their family size; this is related to variations in the adoption of birth control in different societies at different times (Bongaart et al. 1990). There is general agreement that socioeconomic development and organized family planning programs both play significant roles in bringing about changes in reproductive behavior; however identifying independent effects of family planning programs proves more difficult (see United Nations 1979, 1986, and Lloyd and Ross 1989 for a review of this work).

Lapham and Mauldin (1985) showed that it is the combination of improved socioeconomic conditions and greater family planning program effort that leads to the strongest associations with increased use of contraception. These findings were used to demonstrate to economic and social development policymakers that consideration of ways to initiate or improve family planning delivery systems should be an integral part of any development strategy.

Family planning programs have varied widely in their emphasis on demand generation activities and supply-side activities such as increasing contraceptive method choice and using varying service delivery approaches. Despite such differences in their characteristics, Bongaarts, Mauldin, and Phillips (1990) outline certain key issues that are relevant for strengthening program performance in a variety of settings:

1. Passive clinical approaches are less successful than programs that make services available to couples in their villages and home.

2. The quality of services is a crucial but often neglected element of programs; this entails choice among a number of methods, to be well informed about alternative methods, to have competent and caring providers of services, to have follow-up exchanges with knowledgeable program staff.

3. No single formula for program design suits all needs. It is imperative to develop culturally appropriate, sensitive approaches and monitor and adjust programs as a result of lessons learned.

4. Political support for family planning is often critical to establishing strong program effort.

The Matlab Project from rural Bangladesh exemplifies the importance and ultimate success of taking into account all of the above mentioned issues. It is one of the most welldocumented experimental projects on family planning in developing countries as a result of the Demographic Surveillance System (DSS) developed by the International Centre for Diarrhoeal Disease Research, Bangladesh (ICDDR,B). The experimental design of Matlab has allowed researchers to examine the differences between the special services invested in the treatment areas against the standard government services provided in the comparison areas. These areas are similar culturally and socioeconomically allowing researchers to conclude that the Matlab Project has succeeded in raising contraceptive prevalence and reducing child mortality substantially even in an environment that is economically and socially unfavorable to these developments (Nag 1992).

The longitudinal, experimental study design of Matlab has allowed researchers to study a variety of inputs and outcomes as a result of the interventions. The very nature of the study 
design allows researchers to draw conclusions on cause and effect. Unfortunately, this is not always the case in public health research. Although randomized controlled trials are the gold standard, a number of real-world issues are encountered that often prevent the use of randomized trials in public health research; these include feasibility and ethical concerns among others. Randomized controlled trials are "primarily a vehicle for evaluating biomedical interventions, rather than strategies to change human behavior. Altering the norms and behaviors of social groups can sometimes take considerable time...." (Global HIV Prevention Working Group, 2008).

From the available evidence that varies in strength, Bongaarts, Mauldin, and Phillips (1990) estimated that without the effects of family planning programs in the 1970s-1980s, fertility in developing countries would have been 5.4 births per women during 1980 to 1985 rather than the actual 4.2. These program effects reflect the buildup of program strength over the preceding years. Ironically, this success, in combination with increased attention to the AIDS epidemic, has led to reduced funding for contraceptive research and most importantly, investment in family planning services in the mid-1990s (UN Population Fund 2005). And, despite the positive effects that family planning programs have had, in much of the developing world and particularly in sub-Saharan Africa, fertility remains well above the level observed in the developed world, where women average about two births.

\section{INTRODUCTION}

Worldwide, there is a large and empirically verified demand for family planning services to space or limit childbearing. Currently, about 201 million women have an unmet need for modern contraception (PRB 2008), that is, they are sexually active, they want to delay or stop childbearing, and are not using a modern method of contraception. Notably, more than 80 million mistimed or unwanted pregnancies (unintended pregnancies) occur each year worldwide, contributing to high rates of induced abortions, maternal morbidity and mortality, and infant mortality (Cleland et al. 2006). Furthermore, family planning has been found to be an essential approach for countries to achieve their Millennium Development Goals (MDGs), particularly goals four and five for improved child and maternal health outcomes (Cleland et al. 2006; Potts and Fotso 2007; Allen 2007; Moreland 2006). Family planning is a cost-effective public health and development intervention. The cost of averting unwanted births is miniscule compared to the costs to the family and country of unwanted births (Cleland et al. 2006). Few public health interventions are as effective as family planning programs at reducing the mortality and morbidity of mothers and infants and have such a breadth of positive impacts (Cleland et al. 2006; Bongaarts et al. 2009).

In the 1970s-1980s, family planning programs were on the rise, leading to important impacts on increasing voluntary family planning use and reducing fertility in many parts of the world. During this same period, numerous family planning program evaluations were undertaken to demonstrate the impact of demand generation and service delivery improvements on contraceptive use and fertility-related outcomes (Bauman, Viadro, Tsui 1994; Samara, Buckner, Tsui 1996; Cuca and Pierce 1977). Evaluations undertaken in this period included small-scale evaluation efforts to test novel service delivery approaches as well as evaluations of community- and national-level mass media, community-based delivery, and policy change initiatives (Samara, Buckner, Tsui 1996). The family planning evaluations used varying study designs, especially in terms of the outcomes measured, the assumptions required, and the strength of the conclusions (Bertrand, Magnani, Rutenberg 1996). The more rigorous family planning evaluations used randomized experiments (experimental designs), quasi-experiments, and multilevel regression methods. Notably, examining the period through the end of 1992, Bauman (1997) found sixteen family planning evaluations that were considered to be randomized experiments (i.e., random 
assignment of individuals or groups). Thus, while many authors at the time acknowledged the difficulties in undertaking randomized experiments of family planning programs (Cuca and Pierce 1977), Bauman's analysis demonstrated that this was not impossible. That said, even the most widely recognized family planning evaluation, the Matlab study mentioned above, did not randomly assign participating villages; a strong advantage of Matlab, however, was the use of longitudinal data and multivariate analyses to demonstrate program impacts.

Although significant gains have been made since the 1970s, the potential benefits of family planning programs have not been realized for millions of women (Cleland et al. 2006). To revitalize political will and funding for a new era in the promotion of family planning and reproductive health $(\mathrm{FP} / \mathrm{RH})$ services, robust evidence-based strategies must continue to demonstrate research-driven best practices and outline the logistics of implementation. A recent report by the Center for Global Development (CGD) Evaluation Gap Working Group concluded that missed opportunities for the collection and analysis of program impacts have led to continued funding of ineffective and inefficient programs (William 2006). Impact evaluation studies are imperative in providing critical evidence to decision makers on how to effectively spend scarce resources. As a result, the objective of this review is to provide an update on family planning program effectiveness since 1994 when there was less attention and funding for family planning programs. In particular, we a) synthesize recent research on family planning program effectiveness, focusing on experimental and quasi-experimental impact evaluation studies; b) discuss program approaches that are successful (and those that are less successful); c) identify gaps in family planning evaluation research; and d) recommend future research and evaluation directions.

\section{METHODOLOGY}

We undertook a systematic search of journal databases for peer-reviewed articles as well as a companion search of gray literature through funder clearinghouse websites, project websites, and correspondence. In addition, we utilized a 'snowball' sampling approach through searching the reference lists of identified articles. The search strategy included word combinations that incorporated evaluation or outcomes with the following terms: family planning, contraceptive use, child spacing, fertility, unmet need, maternal health, quality of care, private sector family planning services, adolescent pregnancy, unintended pregnancy, abortion, cost effectiveness, male involvement, breastfeeding and lactational amenorrhea method (LAM), and family planning/reproductive health policy.

The inclusion criteria for the review focused on studies of family planning interventions that took place in developing countries, assessed changes in outcomes that are directly attributable to a program (causality), and included the following family planning outcomes of interest: use of family planning services, knowledge and/or attitudes about family planning, discussions around family planning, intentions to use family planning, contraceptive use, unmet need, total fertility rate, unintended pregnancies, and abortion. These outcomes were selected as they provide both short-term and longer term perspectives of family planning program achievements. While the long-term outcomes (fertility, unintended pregnancies, and abortion) are the most important, few evaluations have a long enough follow-up period to observe changes at this level. Thus, using the short-term outcomes provides an understanding of whether programs are on track for achieving their intended impacts; a program that is unable to affect short-term outcomes is unlikely to have long-term impacts. The systematic search covered published and unpublished papers from 1995 to 2008. 
Studies identified were categorized based on their study methodology as experimental designs, quasi-experimental designs, and non-experimental designs. Studies that included experimental designs, that is, the groups or individuals were randomly assigned and quasiexperimental designs with non-random assignment to groups were included in this review. Most of these studies used a pre-test and post-test study design or a panel/longitudinal design. A small number of included studies used a post-test only design with an appropriately defined comparison group. Finally, a few of the included studies were nonexperimental and thus did not include a comparison group but were able to attribute changes in outcomes to program exposure through multivariate analyses.

The studies included in this review meet the above criteria; however, they still vary widely in strength of design and robustness of the findings. For this reason, we further ranked the studies by the methodological quality, creating a rating scale based on the strength of the research design, scope of the study (i.e., ability to generalize results), and the control of confounders and selection bias. As a result, three categories of strength of evidence emerged:

- High - This includes randomized cluster designs that included details on the randomization process and where necessary, controlled for differences in the small number of groups randomized. Also included in this category are studies that randomized individual-level participants; many of these often first randomized sites and then randomized participants within sites. A small number of studies met the high quality criteria by using a longitudinal design with a low loss to follow-up, a long follow-up period, and a comparison group (e.g., Matlab and Navrongo); most of these studies also controlled for differences in groups.

- Medium - Most of the studies in this category used a pre-post test with comparison group design that had a follow-up period of at least six months. All of the studies in this category controlled for possible selection bias between the groups through multivariate analyses. This category also included longitudinal studies without a control group and with low loss to follow-up as well as a small number of studies that used a randomized cluster design but either did not provide details on the randomization process and/or did not control for differences between the small number of intervention and control groups;

- Low - These studies were quasi-experimental designs that often included a prepost test control group design with no control for differences between the groups and/or a very short follow-up period (e.g., $<6$ months). Also in this category are the post-test only comparison group design studies and the longitudinal studies with high loss to follow-up and no comparison group.

Two individuals (the first two authors) independently assessed the studies for inclusion and rated the studies according to the inclusion criteria and above rating scale. The majority of the studies in this review fall into the medium strength of evidence category.

No attempt was made to conduct a meta-analysis and reanalyze the data from the studies, as is done in the Cochrane Collaboration. As noted in the Cochrane Handbook for Systematic Reviews of Interventions, "Public health and health promotion interventions are broadlydefined activities that are evaluated using a wide variety of approaches and study designs. For some questions, the best available evidence may be from non-randomized studies" (Armstrong R et al., 2008). Thus merging study designs and observations from multiple studies would not provide useful information to summarize the varying types of family planning program activities. 
Search results (as of August 2009) yielded 225 studies that consisted of a family planning intervention. Of these, 63 studies met the above methodological criteria for rigor of evaluation. A number of studies were excluded due to their lack of multivariate analysis with non-randomized study designs, focus on reproductive health outcomes other than the family planning outcomes of interest (e.g., HIV prevention programs and youth programs focusing on delayed sexual debut and condom use), or being strictly operations research (e.g., feasibility and acceptability studies) that did not go on to examine population-based family planning and fertility outcomes. Notably, many of the operations research studies were undertaken as part of the FRONTIERS Project led by the Population Council and can be found on the Frontiers Legacy website (http://www.popcouncil.org/publications/ FRONTIERSLegacy/index.asp).

In numerous cases, the interventions consist of various activities using both demand- and supply-side strategies. However, for this review, we have categorized each intervention study as predominantly demand or predominately supply. Forty-two of the included articles are classified as demand-side interventions, while the remaining twenty-one are classified as supply-side interventions. Within the demand generation activities, we further classified programs as mass media, interpersonal communication, and development approaches. The development approaches that included conditional cash transfer programs and a savings and credit program tended to be the most integrated in terms of demand- and supply-side strategies and four out of five of them had high quality evidence (see details below). Among the supply-side interventions, we further classified programs into access, quality, and cost approaches. The one cost approach identified (a voucher program) had features of both a supply-side and a demand generation activity and was classified in the low quality of evidence category.

\section{RESULTS}

Among the 63 studies included, the strength of the evidence varies widely. In particular, among the 42 studies that were in the demand category, 7 were of low quality, 27 were of medium quality, and 8 were considered to be of high quality (see Table 1 for citations by category). Half of the high quality studies were of studies and interventions classified as development approaches - conditional cash transfer programs and a savings and credit program (Stecklov et al. 2007; Steele et al. 2001). Among the remaining high quality demand programs, two were interpersonal communication programs with an instructor/ facilitator (Cabezon et al. 2005; Walker et al. 2006) and two were community-level interpersonal communication programs (Lou et al. 2004; Ross et al. 2007). Among the programs in the supply-side interventions, 8 were considered low quality, 7 were considered medium quality, and 6 were considered high quality. Among those supply-side programs of high quality, three are access/community outreach programs that were undertaken in large demographic surveillance sites (Bangladesh and Ghana), permitting long-term follow-up of a longitudinal sample (Rahman et al. 2001, Sinha 2005, Debpuur et al. 2002). The three others were quality of care/integrated service programs (Bashour et al. 2008; Bolam et al. 1998; Xiaoming et al. 2000), two of which were able to randomize individuals at the clinic level. Notably, the remaining demand-side and supply-side interventions were of medium or low quality but still met the inclusion criteria of being quasi-experimental designs (or having another way to attribute program exposure to outcomes).

Table 2 summarizes the findings of the 63 rigorously evaluated studies included in this review. Generally speaking, the available evidence over the last 15 years suggests that family planning programs have positively impacted individuals' family planning knowledge, attitudes, discussion, intentions, and to a smaller degree, contraceptive use. Seventy-five percent of the studies that measured contraceptive use as an outcome reported positive 
findings for increased contraceptive use or reduced unmet need, while the outcomes of knowledge, attitudes, discussion, and intentions were more commonly found to be significant. Increased service use and changes in fertility-related outcomes were less consistently evident. All of the supply-side interventions that measured fertility outcomes (4 studies - Rahman et al. 2001; Sinha 2005; Debpuur et al. 2002; Sherwood-Fabre et al. 2002) were positive and significant, revealing either a decrease in fertility rates, reduced unintended pregnancies, or a decrease in abortion rates, while only 2 (Askew et al. 2004 (sites A \& C); Cabezon et al. 2005) out of 9 demand-side intervention studies (Rogers et al. 1999; Vernon et al. 2004; Mathur et al. 2004; Stecklov et al. 2007 (included as 3 separate studies - Honduras, Nicaragua, and Mexico); Signorini et al. unpublished, PAA 2009) that measured fertility-related outcomes revealed statistically significant, positive findings on this outcome.

As shown in Table 3, the majority of the evaluation studies reported on interventions that took place in Africa ( $\mathrm{n}=25$ studies), while 21 studies reported on data from Asia, 14 studies from the Americas, 2 study from Eurasia, and 2 studies from the Middle East. ${ }^{1}$

To facilitate synthesis and presentation, studies are presented based on their categorization as demand-side or supply-side interventions below. More detailed summaries of the intervention type, program description, research design/analytic methods, and results may be found in the Appendix.

The next three sections outline specific demand-side approaches that the articles from our review broadly fall within - mass media, interpersonal communication, and development approaches. The subsequent three sections divide the supply-side studies from our review into the three broad supply-side categories - access, quality of care, and cost. The article concludes with a discussion of gaps and directions for future evaluation research.

\section{Demand-side approaches}

Mass media-The central goals of family planning demand-side interventions include changing women's, men's, and couples' knowledge and attitudes about family planning methods, increasing their knowledge of sources of contraceptives, and increasing their use of family planning to meet their fertility desires (Salem et al. 2008). As an intervention, mass media through the radio, television, or print media is an appealing strategy for the promotion of family planning because of its potential reach and ability to address often culturally taboo issues in an entertaining way. The use of media to deliver primarily social development messages has been employed in family planning (FP) and reproductive health (RH) programs for over five decades (Salem et al. 2008). As FP/RH programs have grown and evolved so have the communication approaches. These approaches are referred to by many names such as entertainment-education (EE); edutainment; information, education, and communication (IEC); and behavior change communication (BCC), to name a few (Salem et al. 2008).

Nine articles (Rogers et al. 1999; Kincaid 2000a; Meekers et al. 1997; Meekers et al. 1998; Van Rossem et al. 1999a; Van Rossem et al. 1999b; Kim et al. 2001; Magnani et al. 2000a; Sood et al. 2004) evaluating the impact of mass media interventions that met our inclusion criteria for rigorous evaluation were found in the literature search. Often, in the case of mass media interventions, evaluations must use creative methods to compare those exposed to the intervention to those not exposed given that the programs tend to be full coverage programs. In cases where a comparison group is not feasible, researchers sometimes divide the sample

\footnotetext{
${ }^{1}$ The total does not equal 63 studies because the Brieger 2001 article reports on data from Nigeria and Ghana.
} 
into groups based on exposure to the various components of the intervention. Comparing the groups based on exposure experience or extent of exposure, controlling for background differences, provides researchers with an opportunity to measure dose response effects on the fertility or family planning outcomes of interest (Kincaid 2000a; Magnani et al. 2000a; Sood et al. 2004). Given these methodological challenges with evaluating mass media approaches, all but one of these studies were considered to be of medium quality; this last study was considered of lower quality because it used a post-test only comparison group design (Sood et al., 2004).

Of the nine mass media intervention evaluations we reviewed, results usually focused on short-term outcomes such as changes in knowledge, attitudes, beliefs, and discussion patterns either between partners or between parents and their children. Few behavioral outcomes were measured. However, when behavioral outcomes such as contraceptive use were measured among the study population, results were positive (Rogers et al. 1999; Kincaid 2000a). Most positive behavioral results emerged from studies where mass media was combined with other intervention components, such as social marketing (Meekers et al. 1998; Van Rossem et al. 1999a; Van Rossem et al. 1999b) or interpersonal communication interventions (Kim et al. 2001; Magnani et al. 2000a; Sood et al. 2004).

In Tanzania, Rogers and colleagues (1999), which was considered to be a medium quality study, measured married women's use of contraceptives as a result of exposure to an entertainment-education radio soap opera, "Twende na Wakati” (Let's Go with the Times). The authors used a quasi-experimental design since the soap opera was broadcasted on seven mainland stations of Radio Tanzania and not on the eighth station covering the Dodoma area. While the seven stations were broadcasting the soap opera twice a week, the Dodoma area station was broadcasting locally produced programs at the same time.

Consequently, it was able to serve as the comparison site. In addition to triangulating a number of different data sources, the authors used a repeat cross-sectional design, in which they surveyed individuals in the same 35 wards in two regions of the Dodoma comparison area and seven regions in the treatment area at one-year intervals from 1993 to 1997. The authors found that all statistical tests supported a significant effect of exposure to "Twende na Wakati” from 1993 to 1995 on married women's use of contraceptives. As a result of the positive findings from the 1993-1995 analysis, Radio Tanzania began broadcasting the soap opera in the Dodoma area. The authors found this statistically significant effect between exposure to the soap opera and contraceptive use of married women replicated in the Dodoma comparison area from 1995 to 1997.

Interpersonal communication-Interpersonal communication approaches including one-on-one discussions, small-group sessions, and facilitator-led curriculum-based programs are another demand-side strategy used to influence knowledge, attitudes, intentions, and behaviors related to FP and RH. Interpersonal communication interventions take place in varying settings including schools, workplaces, and the community. These interventions are often facilitated by peers, teachers, or expert trainers. Twenty-eight articles using interpersonal communication approaches met our inclusion criteria. Of these articles, 11 reported on peer-led interventions (Agha et al. 2004; Magnani et al. 2000b; Brieger et al. 2001; Speizer et al. 2001; Cartagena et al. 2006; Askew et al. 2004; Diop et al. 2004; Vernon et al. 2004; Bhuiya et al. 2004; Mathur et al. 2004; Ozcebe et al. 2003); 12 were instructor/facilitator-led (Cabezon et al. 2005; Eggleston et al. 2000; Magnani 2001; Mbizvo et al. 1997; Murray et al. 2000; Martiniuk et al. 2003; Mba et al. 2007; Rusakaniko et al. 1997; Shuey et al. 1999; Stanton et al. 1998; Walker et al. 2006; FOCUS/CARE International - Cambodia 2000); and, 5 were community-based (Levitt-Dayal et al. 2001; Erulkar et al. 2004; Lou et al. 2004; Tu et al. 2008; Ross et al. 2007). 
Within these categories, the overwhelming majority were of medium quality with the exception of two of the peer-led interventions that were lower quality (Cartagena et al. 2006; Ozcebe et al. 2003); two of the instructor/facilitator led that were of high quality (Cabezon et al. 2005; Walker et al. 2006); two of the instructor/facilitator led of low quality (Mba et al. 2007; Shuey et al. 1999); two of the community-based that were high quality (Lou et al. 2004; Ross et al. 2007); and one of the community-based that was low quality (Levitt-Dayal et al. 2001). Notably, none of the articles compared the different types of facilitators to help inform whether one approach is more effective than another. However, Table 2 reveals that the peer-led and adult-led intervention studies had similar outcomes.

The interpersonal communication interventions almost exclusively targeted adolescents and young adults (the age range included 10 year olds to 26 year olds), and the evaluations all included short-term outcomes (knowledge, attitudes, and beliefs) and only a few included behavioral outcomes (contraceptive use and unintended pregnancies). Most studies (86\% 18 studies out of 21) reported improved knowledge and attitudes, while about two-thirds of the studies (12 studies out of 19) measuring family planning use found a positive effect (Magnani et al. 2000b; Brieger et al. 2001; Speizer et al. 2001; Askew et al. 2004; Murray et al. 2000; Stanton et al. 1998; Walker et al. 2006; Levitt-Dayal et al. 2001; Erulkar et al. 2004; Lou et al. 2004; Tu et al. 2008; Ross et al. 2007) and half of those measuring fertility outcomes ( 2 studies out of 4 ) led to declining fertility (Askew et al. 2004; Cabezon et al. 2005).

Askew and colleagues (2004) compared three intervention sites in the Western Providence of Kenya. Interventions to create a supportive environment at the community level and strengthen the health system's ability to meet the reproductive health information and service needs of adolescents were introduced into Site A locations. Site B locations consisted of the same intervention activities as in Site A plus an in-school life-skills and development curriculum and parent sensitization, so that the additional effect of educating school children on reproductive health issues could be assessed. Site $\mathrm{C}$ locations were identified as comparison sites, where no interventions were introduced. This was considered to be a medium quality study. For most socio-demographic characteristics, there were no differences, between the sites nor between baseline and endline cross-sectional characteristics. Where differences did occur for a characteristic, it was taken into account in the analysis and interpretation of the findings. This indicates that any differences in measures of the key indicators found between baseline and endline are most likely due to the influence of the interventions themselves. Among the never married girls living in Site B there was no change over time in terms of experiencing pregnancy, with about one quarter of sexually active girls reporting a pregnancy. In intervention Site A and the comparison site, however, large and significant reductions in pregnancy were reported over time. This may be a result of the fact that approval of contraception and condom use improved in the comparison site and in Site A but not in Site B. This finding is particularly interesting because Site B, which offered exposure to the largest number of program components, had the least effect.

Alternatively, Cabezon and colleagues (2005), in their high quality study, found that a school-based intervention taught by teachers had a protective effect in preventing unintended pregnancies. Three cohorts of first year high school students were enrolled in a randomized control trial in which some students received no intervention and other students received the TeenSTAR abstinence-centered sex education program which consisted of 14 units taught over a school year. The cohorts represent the years 1996, 1997, and 1998; the 1996 cohort did not experience any intervention program. No interventions were received by any of the cohorts during their second, third, or fourth years. All cohorts were followed up for four years; pregnancy rates were recorded and subsequently contrasted in the 
intervention and control groups. Pregnancy rates for the intervention and control groups at four year follow-up in the 1997 cohort were $3.3 \%$ and $18.9 \%$, respectively; while pregnancy rates for the intervention and control groups at four year follow-up in the 1998 cohort were $4.4 \%$ and $22.6 \%$, respectively. The pregnancy rate for the 1996 cohort that was not exposed to the program was $14.7 \%$. The differences between intervention and control group by cohort were statistically significant demonstrating an impact of the TeenSTAR program using a high quality study design.

Development approaches-The development approaches, which included four conditional cash transfer programs and a savings and credit program, all focused on intermediate and long-term behavioral outcome indicators, such as contraceptive use and fertility. Four out of five of these studies were of high quality (Stecklov et al. 2007 counting for three studies; Steele et al., 2001) and the remaining one was of medium quality (Signorini et al. unpublished, PAA 2009). In the case of Stecklov and Signorini, they used population-level secondary data for the evaluation. In particular, by relying on large, nationally representative surveys that are infrequently available, the authors were able to examine longer-term outcomes than what is usually available immediately following the intervention activities. Stecklov and colleagues (2007) explored the possible association between conditional cash transfer (CCT) programs in three Latin American countries (Mexico, Nicaragua, and Honduras) and fertility. The authors compared three sets of panel data from experimental CCT programs in these countries to assess the impact of these programs on childbearing. Each program first identified a set of communities eligible for the program and then randomly assigned them into control and treatment groups. The treatment groups were provided payments conditional on the household's behavior, such as enrolling children into public schools, getting regular check-ups at the doctor's office, and receiving vaccinations.

Findings, based on difference-in-difference models, show that the program in Honduras, which inadvertently created large incentives for childbearing, may have raised fertility by between 2 and 4 percentage points. The Honduras program created incentives by establishing different targeting and eligibility criteria and transfer amounts. For example, the Honduras' Family Allowance Program (PRAF, after its Spanish acronym) allowed parents to join or obtain increased benefits by bearing children after the program had begun (Stecklov et al. 2007). The CCT programs in the two other countries, Mexico and Nicaragua, did not have the same unintended incentives for childbearing; however, they also did not have a net impact on fertility (Stecklov et al. 2007). The data were more positive when examining contraceptive use, where it was measured. The data from the PROGRESA program in Mexico revealed significant increases in contraceptive use, while the Nicaragua data illustrated an increase, albeit one that was not significant. Similarly, an evaluation of a similar conditional cash transfer project in Brazil, called Bolsa Familia Program, was found to have no impact on the fertility of program beneficiaries (Signorini et al. unpublished, PAA 2009).

Likewise, evidence from a high quality evaluation of a Save the Children USA program examining the characteristics of women who choose to join a women's savings or a credit group in rural Bangladesh and the impact of their participation on contraceptive use revealed mixed results (Steele et al. 2001). The credit approach required more stringent criteria for membership based on credit worthiness, an admission fee, and there were individual and group expenses for meeting rooms. In addition, the group funds were managed by a credit officer who collects weekly savings and loan payments to deposit at a government bank. The savings groups were more autonomous than the credit groups, and set their own rules with regard to frequency of meetings, savings contributions by members, size of group, and how group savings were managed. To evaluate the impact of the credit program, the authors 
compared credit members with eligible nonmembers in the same village communities. The savings group members were compared with eligible nonmembers in the same village communities as well as in village communities in which the savings program was not introduced. The use of a longitudinal design for this evaluation controlled for two types of endogeneity that often threatens evaluation research: self selection and non-random program placement (Steele et al. 2001). Increased contraceptive use was found among participants of the credit program but not among participants of the savings group.

\section{Supply-side approaches}

The overarching strategy of successful supply-side family planning programs is to make contraceptive methods as accessible as possible to clients in a good quality, reliable fashion. This includes offering a wide range of affordable contraceptive methods, making services widely accessible through multiple service delivery channels, making sure potential clients know about services, following evidence-based technical guidelines that promote access and quality, and providing client-centered services (Richey et al. 2008). These types of supplyside interventions ensure that women and couples are able to effectively use family planning when the need arises. Understanding which supply side interventions lead to increased contraceptive use and reductions in unmet need and unintended pregnancy is important for making recommendations to program managers and policy makers on how to expend finite resources. Much of the research to date on supply-side interventions has been undertaken through operations research that has generally focused on outcomes such as improved service quality, increased client satisfaction, and increased service use. Fewer recent evaluation studies of supply-side strategies examine whether changes in family planning availability, accessibility, quality, and costs lead to increased contraceptive use and reduced unintended pregnancy at the population level. The studies that we sought for this assessment of rigorous evaluations did just this; they examined the impact of supply-side activities on fertility and family planning outcomes. In total, we found twenty-one articles that evaluated the population-level impact of supply-side interventions.

Access-Nine studies focusing on issues of accessibility met our inclusion criteria; three of these (two with medium quality and one with low quality) evaluated the impact of fractional social franchising programs (Agha et al. 2007, Hennink and Clements 2005, Babalola et al. 2001) and six focused on community-based distribution or outreach programs (high quality: Rahman et al. 2001, Sinha 2005, Debpuur et al. 2002; medium quality: Phillips et al. 1996; low quality: Douthwaite et al. 2005, Kincaid 2000b). Social franchising typically entails the creation of networks of private medical practitioners (doctors, nurses, midwives, pharmacists) that offer a standard set of services at lower costs under a shared brand name. Franchise members are offered training, commodity advertising, inter-franchise referrals, a branding that shows high-quality standards, and other benefits. Fractional social franchises are businesses that add a franchised service or product to the existing operations (LaVake 2003). Among the three studies that used this approach, one with medium quality and one with low quality (Hennink and Clements 2005; Babalola et al. 2001) had a significant effect on family planning outcomes in the intended direction, while the Agha et al. 2007 article found a marginally significant effect on current use of family planning ( $p=$. 067). None measured an effect on fertility-related outcomes. Similarly, those that examined knowledge, attitudes, and intentions also demonstrated positive effects. One study, however, that examined whether fractional social franchising leads to increased service use in Nepal failed to show the hypothesized effect and showed only a marginally significant effect for contraceptive use (Agha et al. 2007). This may be a result of the fact that clients had other sources of reproductive health services available to them that they felt comfortable using; $10-12 \%$ of the population in the intervention district went to a medical store/pharmacy for 
reproductive health services. In addition, the project was implemented for less than a year (Agha et al. 2007).

Hennick and Clements (2005) found that the introduction of new family planning clinics in urban Pakistan resulted in increased knowledge of family planning methods, distinct effects on contraceptive uptake, and decline in unmet need. However, the impacts were different by provinces, which represent different cultural contexts. The new clinics in Sargodha and Gujranwala in the Punjab province contributed to a significant decline in unmet need for family planning; most of this change was comprised of a decline in unmet need for limiting births. In the more culturally conservative cities of Hyderabad and Shikarpur in the Sindh province, the operation of the new clinics led to no reduction in overall unmet need but led to increases in the demand for family planning. This study highlights the importance of taking into account the socio-cultural context of the study location.

Among the six community outreach/distribution studies, positive findings were found for all outcomes measured, including three studies that measured fertility related outcomes. The three studies that measured fertility outcomes were all of high quality and used longitudinal study designs - Matlab and Maternal and Child Health-FP Extension projects in Bangladesh (Rahman et al. 2001; Sinha 2005) and Navrongo project in Ghana (Debpuur et al. 2002) over long follow-up periods. This reflects the fact that it is often difficult to report on changes in fertility-related outcomes in the absence of datasets that cover a long period of time. In addition, the Matlab and Maternal and Child Health-FP Extention and Navrongo projects include a combination of demand- and supply-side activities, which may explain the positive FP and fertility outcomes.

These long follow-up periods also provided the time necessary to compare different intervention approaches on the outcomes of interest. For example, Debpuur and colleagues (2002) examined approaches to mobilizing Ghana's Ministry of Health outreach program and compared this with mobilizing traditional community-based organizations as well as mobilizing both sectors simultaneously. Their study had four arms: a comparison site with no intervention, a nurse outreach only site, a traditional community organization (zurugelu) site, and a combined zurugelu plus nurse outreach site. They found that contraceptive use remained the same when analyzing the effects of the approaches separately. However, when examining the combined zurugelu plus nurse outreach approach, they found that contraceptive use increased significantly and fertility decreased significantly.

Quality of care-Programs that seek to improve quality of services often focus on the various components of quality as defined by Bruce (1990) in her seminal article. These include choice of methods, information given to users, technical competence, interpersonal relations, follow-up or continuity mechanisms, and appropriate constellation of services. Quality is inconsistently defined across different studies, and between different stakeholders. This makes it difficult to draw larger conclusions about studies that seek to improve family planning service quality.

Eleven articles to improve quality of care met the inclusion criteria for this review. One article of lower quality focused on increased method options (Khan et al. 2004 - introducing emergency contraceptive pills into the method mix); another of lower quality focused on client provider interactions (Nawar et al. 2004). Nine reported on various quality improvement approaches (high quality: Bashour et al. 2008, Bolam et al. 1998, Xiaoming et al. 2000; medium quality: Khan et al. 2008, Kunene et al. 2004, Sherwood-Fabre et al. 2002, Sanogo et a. 2003; low quality: Varkey et al. 2004, Speizer et al. 2004). 
Studies that sought to improve quality were not consistently successful; six studies (Sanogo et a. 2003; Xiaoming et al. 2000; Speizer et al. 2004; Bolam et al. 1998; Khan et al. 2008; Varkey et a. 2004) out of ten revealed a significant increase in contraceptive use. Five of the seven integrated service delivery studies focused on postpartum contraceptive use (Bashour et al. 2008; Bolam et al. 1998; Khan et al. 2008; Kunene et al. 2004; Varkey et al. 2004), and of these, three reported significant results (Bolam et al. 1998; Khan et al. 2008; Varkey et a. 2004). The one integrated service delivery study that provided family planning to postabortion clients in Russia (Sherwood-Fabre et al. 2002) found a reduction in abortion rates but no corresponding increase in contraceptive use. The authors explained that this situation may be a result of the fact that the intervention was unevenly implemented; the survey indicated that there were many missed opportunities to reinforce and personalize the family planning information that women received. In addition, it was found that there was an increase in the proportion of unintended pregnancies that resulted in live births during the study period; this affected the abortion rates (Sherwood-Fabre et al. 2002). Finally, one study investigated the impact of integrating an HIV prevention intervention into a wellestablished family planning network of services in China. The authors found that at 12months follow-up, significantly more respondents from the experimental sites were using condoms as their main contraceptive method ( $\mathrm{p}<.05$ ) (Xiaoming et al. 2000) as compared to in the comparison sites.

The feasibility of male involvement in antenatal care (ANC) counseling sessions and the effectiveness of their involvement in postpartum contraceptive use was evaluated by two studies - one of medium quality from South Africa (Kunene et al. 2004) and one of lower quality from India (Varkey et al. 2004). In KwaZulu-Natal, South Africa, the study team matched facilities by size and rural/urban status and then randomly assigned six clinics as the intervention sites and six as the comparison sites. Implementing joint couple counseling was challenging for this program because the population being served was mostly unmarried. One-third of the couples invited attended the joint counseling sessions, and communication among them was reported to improve with male partners more likely to provide support in the event of pregnancy complications; however, postpartum family planning use and overall risk behavior did not change (Kunene 2004). The other study that examined male involvement had positive effects; however, did not randomly assign sites or control for differences between the groups (Varkey et al. 2004).

Cost-The issue of cost of family planning methods is often discussed as both a supplyside and demand-side issue. From the supply-side perspective, the direct cost of a family planning method is seen as a barrier to use. From the demand-side perspective, many family planning programmers and advocates have pointed out that there are many indirect costs associated with access to family planning, such as large, unofficial payments to staff and long waiting times to see service providers; each of these affects demand (Ensor and Cooper 2004).

Only one article evaluating a cost-based intervention was found that met our inclusion criteria. Although Meuwissen and colleagues (2006) used a quasi-experimental design to evaluate the impact of a competitive voucher pilot program on adolescents' use of sexual and reproductive health care (SRHC) services and contraceptives in urban Managua, Nicaragua, the authors used a post-test only design so the study design is considered lower quality. Self-administered questionnaires were distributed randomly among female adolescents 3 to 15 months after the vouchers had been distributed in their area. The voucher receivers were considered the intervention group to be compared at a group level with the control group, the non voucher receivers. Voucher receivers demonstrated significantly higher use of SRHC services and knowledge of contraceptives and sexually transmitted infections compared with non-receivers. There was no change in overall contraceptive use 
between the two groups - receivers and non-receivers. However, effects were modified by place of survey - school versus neighborhood. Focus group discussions and interviews with adolescents during the intervention suggest that the factors that contributed to the success of the voucher program were the removal of practical obstacles (e.g., financial, the need to make an appointment, the lack of information on clinic location, and opening times) plus the guarantee of confidential access to a service provider of their choice. These results reveal the interplay between demand-side and supply-side barriers.

\section{DISCUSSION}

The available evidence on the effectiveness of family planning interventions in developing countries over the last fourteen years reveals a positive picture with no one size fits all approach. Both demand- and supply-side interventions led to improvements in knowledge, attitudes, discussion of family planning and sexuality, and intentions to use family planning. Results were less consistent in terms of effects on fertility and family planning outcomes.

The examination of mass media interventions illustrated positive results on contraceptive use and/or unmet need, while the wealth of interpersonal communication interventions less consistently demonstrated these effects. A notable example is the quasi-experimental study by Rogers and colleagues (1999) that showed a significant effect of exposure to an entertainment-education radio soap opera on contraceptive use by married women, which led to the scale-up of the program nationwide throughout Tanzania.

Only two (Askew et al. 2004; Cabezon et al. 2005) out of the four studies from the interpersonal communications category that measured fertility related outcomes found significant reductions in unintended pregnancies. For example, Askew and colleagues (2004) found differential effects by exposure arm such that those with the greatest exposure had the least fertility and family planning impacts. Askew and colleagues acknowledged that their multi-sectoral approach reached adolescents with reproductive health information; however, they cautioned that the findings need to be interpreted with care in light of the fact that the community-based intervention was more intensively implemented in varying sites. They also pointed to the fact that the teachers who implemented the school-based intervention were more comfortable with providing the abstinence messages than the safer sex messages to their students. These challenges are representative of similar challenges experienced by all programs working with adolescents on issues of sexual and reproductive health.

Contrary to Askew's findings, Cabezon and colleagues (2005) revealed a protective effect in preventing unintended pregnancies from a school-based intervention taught by teachers. The authors reported that the success of their teacher-led program was due to the accurate and comprehensive information provided and the focus on developing assertiveness and negotiation skills. The authors also acknowledged that the implementation of the program over an academic year was ideal and found the teachers to be effective implementers of the program. This example along with the example provided by Askew highlights the importance of the program facilitators' comfort with the subject matter and their commitment to the program.

Once demand for contraceptive use is achieved, it is imperative that the supply is readily available and accessible. Our review found that supply-side interventions that addressed access to family planning led to positive effects on family planning use, whereas improved quality less consistently showed positive effects on family planning behaviors. Notably, few studies measured fertility-related outcomes such as reduced unintended pregnancies and abortions; however, the supply-side intervention studies that did measure fertility showed 
the most consistent and positive findings, generally using the strongest study designs (Khan et al. 2008; Debpuur et al. 2002; Rahman et al. 2001; Sherwood-Fabre et al. 2002).

It is also notable that even in some places where the findings were positive; results were not necessarily consistent across different locations or target groups. For example, the voucher program in urban Nicaragua found different effects among school-going youth and youth who participated from community-based sites (Meuwissen et al. 2006). Hennick and Clements (2005) reported differential changes in unmet need as a result of introducing new family planning clinics in two culturally distinct provinces of Pakistan. Debpuur and colleagues (2002) found a significant increase in contraceptive use and decrease in fertility when they examined the combined approach of two different community-based outreach interventions in contrast to when they analyzed the effects of the interventions separately.

Although the findings presented in this review categorized studies as demand-side and supply-side interventions, a small number of studies explicitly included a multi-component approach, such as undertaking mass media and interpersonal communication (Kim et al. 2001; Magnani et al. 2000a; Sood et al. 2004), mass media and social marketing (Meekers et al. 1998; Van Rossem et al. 1999a; Van Rossem et al. 1999b), fractional social franchising with strong media promotional presence (Agha et al. 2007; Babalola et al. 2001), and fractional social franchising and community-based outreach (Hennick and Clements 2005). These studies generally found positive family planning outcomes and, when measured, positive fertility outcomes as well. The importance of multi-component programs has been demonstrated in other reviews that have examined rigorous evaluations of adolescent reproductive health programs (Speizer et al. 2003; Ross et al. 2006).

It is also worth noting where the evidence was weak or non-existent in this review. While male involvement programs are becoming increasingly important in the international family planning arena, there is limited evidence on the effectiveness of this approach on population-based fertility and family planning outcomes. A small number of operations research studies have been undertaken and reveal that approaches to increase male involvement in prenatal and postpartum care lead to increased attendance at these critical events; these studies with medium to low quality of quasi-experimental design, however, have shown mixed results in regards to behavioral outcomes such as contraceptive use and unintended pregnancies (Kunene et al. 2004; Varkey et al. 2004).

In addition to the limited evidence provided on evaluations of male involvement programs, only one study of lower quality (Meuwissen et al. 2006) was found that examined the effect of a voucher program on increased contraceptive use behaviors; this is an important gap because voucher programs are an increasingly popular approach in public health programming. Several developing countries, with international donor support, are considering or in the process of implementing a voucher program (examples include India, Tanzania, Uganda, Kenya and Bangladesh) (Arur et al. 2009). However, more research is needed to inform the design and expansion of voucher programs throughout the developing world. Important areas for further voucher studies include: impact evaluations, cost and cost-effectiveness studies, and the effect of using technology to simplify implementation and reduce overhead costs. Vouchers are a relatively untested approach in low-income countries. Although results from these early experiences are positive, there is a clear need for rigorous research that can conclusively establish that voucher programs can increase coverage and use of FP/RH and other health services among underserved target populations (Arur et al. 2009). 


\section{Limitations}

There are a number of limitations to this type of systematic review. First, depending on the level of depth provided in each study, it is not always possible to extrapolate the features of each intervention and the corresponding evidence as to which components were the most effective. Second, most studies of multi-component programs examined overall program effects and did not separate out the effects of the different components. Third, the majority of studies considered were written in English although the authors also reviewed studies in French. Fourth, as expected for the outcomes of interest, most of the studies were based on self-reported sexual and health-seeking behaviors; previous studies have demonstrated potential biases of self-reported behaviors (Curtis and Sutherland 2004). Fifth, service utilization statistics were included but only as a complement to individual level data; this limited the inclusion of most of the operations research studies. Sixth, there is likely to be a publication bias with this type of review whereby studies with positive findings are more likely to be published (and found) whereas studies with non-significant or negative findings are unavailable. Finally, given the diversity in study methods and implementation strategies, it was not possible to do a formal meta-analysis that joins the samples and compares the odds ratios. Therefore, a limitation of this study is that while all studies included met the experimental or quasi-experimental (or another form of attribution) criteria, there was still variability across the rigor of the studies. We have categorized the identified studies into three quality categories: low, medium, and high to help clarify these types of distinctions across the multiple approaches. Where appropriate, we have identified which findings come from those studies with a higher study quality (e.g., are from a randomized cluster design and/or use longitudinal data with a comparison group to determine attribution).

\section{Conclusions}

In spite of limited funding for family planning programs during the period 1995 to 2008, this systematic review reveals that both demand- and supply-side interventions that have been rigorously evaluated have been found to be generally successful in increasing knowledge, attitudes, beliefs, and discussions around family planning as well as increasing contraceptive use. These impacts are often a result of programs that have taken into account the importance of various approaches to reaching women and couples with family planning products and services, providing quality information and service delivery, addressing cultural norms and barriers to contraceptive use, and seeking community support.

That said, a number of gaps and directions for future research have also been identified. In particular, there is a need to undertake evaluations of broader development approaches and supply-side interventions measuring population-level outcomes (beyond operations research) and their long-term impacts on family planning and fertility. Likewise, there is a need for more research around the impact of male involvement, integrated services, publicprivate partnerships, and voucher programs, especially in light of the recent push and funding for these approaches. There is also a need for information on the effectiveness and cost-effectiveness of alternative implementation approaches for both demand and supplyside interventions. For example, there are gaps in our understanding of the impact of a peerled versus instructor or facilitator led program as well as gaps in the understanding of intervention costs and the comparison of costs for alternative implementation approaches.

Most evaluations are of small-scale interventions and implemented over relatively brief periods of time (often a pilot test). There is little evidence on the long-term behavioral effects of the interventions that would provide us the evidence required to make decisions about scale-up or replication. The strongest evidence to support reaching long-term fertility measures comes from long-standing longitudinal studies, such as Maternal and Child Health-FP Extension projects in Bangladesh (Rahman et al. 2001; Sinha 2005) and the 
Navrongo project in Ghana (Debpuur et al. 2002). This reflects the need for long-term follow-up in measuring and observing such changes in fertility-related outcomes. A number of Demographic Surveillance Systems coordinated through the INDEPTH network (http:// www.indepth-ishare.org/) are now currently available and could possibly serve as data for these types of long-term evaluations of existing models in varying sites.

Furthermore, lacking in most studies is an assessment of the differential impact of interventions across target audiences. For example, it is important to consider the impacts of programs on the populations most in need of services, such as high risk subgroups, migrants, and the urban poor, to name a few. The examination of differential impacts by subgroups was rarely examined within the studies found. More specific information on the actual beneficiaries of interventions is still needed by policy makers wishing to target scarce resources to those most in need. The study by Hennick and colleagues (2005) highlights the importance of not only understanding the cultural backgrounds of the various populations that are being studied, which helps in explaining the different results in unmet need and where these two distinct populations are in terms of acceptance and intentions to use family planning methods, but also the importance of looking at the socio-demographic characteristics of beneficiaries. They found that women who live outside of the catchment areas are often married with four or more children and are of low socioeconomic status. They also found that young (aged 16-19), poor women who are separated or unmarried were seeking services from outside the catchment areas. These findings reveal that women who are poor were willing to travel some distance to obtain services for which they pay fees.

The findings of this review reveal that all of the intervention approaches have some benefit at least on short-term outcomes. The main program approaches that led to increases in contraceptive use included development approaches and supply-side interventions. Whether the other approaches did not have an effect or did not measure one is a different issue. Notably, only a small number of studies had an impact on fertility outcomes; most of these were high quality studies of supply-side approaches working in supportive, long-term settings using multi-component, integrated programs. As interventions are designed, it is imperative that planning goes into monitoring and evaluating the activities, so that programs can be refined and lessons learned can be shared widely. Particular attention needs to be paid to undertaking rigorous impact evaluations that can attribute program activities to changes in outcomes of interest. Randomized controlled trials will not be feasible for most FP program activities; thus evaluators need to identify alternative study designs (quasiexperimental; longitudinal) that are appropriate for the varying settings where programs are being implemented (Victora, Habicht, and Bryce, 2004). This attention to rigor of family planning evaluations will increase accountability, improve program decision making, and in the end, improve maternal and infant health outcomes.

\section{Acknowledgments}

This work was made possible with support from the Bill \& Melinda Gates Foundation for the Measurement, Learning \& Evaluation (MLE) Project for the Urban Reproductive Health Initiative. The authors' views expressed in this publication do not necessarily reflect the views of the donor, the Bill \& Melinda Gates Foundation.

\section{References}

Agha S. A Quasi-Experimental Study to Assess the Impact of Four Adolescent Sexual Health Interventions in Sub-Saharan Africa. International Family Planning Perspectives. 2002; 28(2):67118.

Agha S, Van Rossem R. Impact of a school-based peer sexual health intervention on normative beliefs, risk perceptions, and sexual behavior of Zambian adolescents. Journal of Adolescent Health. 2004; 34(5):441-452. [PubMed: 15093801] 
Agha S, Karim AM, Balal A, Sosler S. The impact of a reproductive health franchise on client satisfaction in rural Nepal. Health Policy and Planning. 2007; 22:320-328. [PubMed: 17644555]

Allen R. The role of family planning in poverty reduction. Obstetrics and Gynecology-New York. 2007; 110(5):999.

Armstrong, R.; Waters, E.; Doyle, J., editors. Chapter 21: Reviews in health promotion and public health. Higgins, JPT.; Green, S., editors. Cochrane Handbook for Systematic Reviews of Interventions. Version 5.0.1. The Cochrane Collaboration; 2008. [updated September 2008], Available from www.cochrane-handbook.org

Arur, A.; Gitonga, N.; O’Hanlon, B.; Kundu, F.; Senkaali, M.; Ssemujju, R. Insights from Innovations: Lessons from Designing and Implementing Family Planning/Reproductive Health Voucher Programs in Kenya and Uganda. Bethesda, MD: Private Sector Partnerships-One project, Abt Associates Inc; Nov. 2009

Askew, I.; Chege, J.; Njue, C.; Radeny, S. A Multi-sectoral Approach to Providing Reproductive Health Information and Services to Young People in Western Kenya: Kenya Adolescent Reproductive Health Project. Washington, DC: FRONTIER, Population Council; 2004 Jun.

Babalola S, V C, Brown J, Traore R. The impact of a regional family planning service promotion initiative in sub-Saharan Africa: evidence from Cameroon. International Family Planning Perspectives. 2001; 27(4):186-193.

Bashour HN, Kharouf MH, Abdulsalam AA, El Asmar K, Tabbaa MA, Cheikha SA. Effect of postnatal home visits on maternal/infant outcomes in Syria: a randomized controlled trial. Public Health Nurs. 2008; 25(2):115-125. [PubMed: 18294180]

Bauman KE, Viadro CI, Tsui AO. Use of true experimental designs for family planning program evaluation: Merits, problems and solutions. International Family Planning Perspectives. 1994; 20(3):108-113.

Bauman KE. The effectiveness of family planning programs evaluated with true experimental designs. American Journal of Public Health. 1997; 87(4):666-669. [PubMed: 9146451]

Bertrand, J.; Magnani, R.; Rutenberg, N. Evaluating family planning programs: with adaptations for reproductive health. Chapel Hill, NC: The EVALUATION Project; 1996.

Bhuiya, I.; Rob, U.; Chowdhury, JH.; Rahman, L.; Haque, N.; Adamchak, S., et al. Improving adolescent reproductive health in Bangladesh. Washington, DC: FRONTIER, Population Council; 2004.

Bolam A, Manandhar DS, Shrestha P, Ellis M, Costello AMdL. The effects of postnatal health education for mothers on infant care and family planning practices in Nepal: a randomised controlled trial. BMJ. 1998; 316(7134):805-811. [PubMed: 9549449]

Bongaarts J, Mauldin WP, Phillips J. The Demographic Impact of Family Planning Programs. Studies in Family Planning. 1990; 21(6):299-310. [PubMed: 2075620]

Bongaarts J. Fertility transitions in the developing world: progress or stagnation. Studies in Family Planning. 2008; 39(2):106-110.

Bongaarts J, Sinding S. A response to critics of family planning programs. International perspectives on sexual and reproductive health. 2009; 35(1):39. [PubMed: 19465347]

Brieger WR, Delano GE, Lane CG, Oladepo O, Oyediran KA. West African youth initiative: outcome of a reproductive health education program. Journal of Adolescent Health. 2001; 29(6):436-446. [PubMed: 11728893]

Buvinic, M.; Médici, A.; Fernández, E.; Torres, AC. Gender Differentials in Health. In: Jamison, DT.; Breman, JG.; Measham, AR.; Alleyne, G.; Claeson, M.; Evans, DB.; Jha, P.; Mills, A.; Musgrove, P., editors. Disease Control Priorities in Developing Countries. 2. New York: Oxford University Press; 2006. p. 195-210.

Cabezón C, Vigil P, Rojas I, Leiva ME, Riquelme R, Aranda W, et al. Adolescent pregnancy prevention: an abstinence-centered randomized controlled intervention in a Chilean public high school. Journal of Adolescent Health. 2005; 36(1):64-69. [PubMed: 15661598]

Cartagena RG, Veugelers PJ, Kipp W, Magigav K, Lain LM. Effectiveness of an HIV prevention program for secondary school students in Mongolia. Journal of Adolescent Health. 2006; 39:925.e9-925.e16. [PubMed: 17116526] 
Casterline JB, Sinding SW. Unmet Need for Family Planning in Developing Countries and Implications for Population Policy. Population and Development Review. 2000; 26(4):691-723.

Cleland J, Bernstein S, Ezeh A, Faundes A, Glasier A, Innis J. Family planning: the unfinished agenda. Lancet. 2006; 368(9549):1810-1827. [PubMed: 17113431]

Cuca, R.; Pierce, CS. Experiments in family planning: Lessons from the developing world. Washington, DC: The World Bank; 1977.

Curtis SL, Sutherland EG. Measuring sexual behaviour in the era of HIV/AIDS: The experience of Demographic and Health Surveys and similar enquiries. Sexually Transmitted Infections. 2004; 80(Suppl II):ii22-ii27. [PubMed: 15572636]

Debpuur C, Phillips JF, Jackson EF, Nazzar A, Ngom P, Binka FN. The impact of the Navrongo Project on contraceptive knowledge and use, reproductive preferences, and fertility. Stud Fam Plann. 2002; 33(2):141-164. [PubMed: 12132635]

Diop, N.; Bathidja, H.; Touré, ID.; Dieng, T.; Mané, B.; RamaRao, S., et al. Improving the Reproductive Health of Adolescents in Senegal. Washington, DC: FRONTIER, Population Council; 2004 Dec.

Donaldson, PJ. Nature against Us: The United States and the World Population Crisis, 1965-1980. Chapel Hill, NC: University of North Carolina Press; 1990.

Donaldson PJ, Tsui AO. The International Family Planning Movement. Population Bulletin. 1990; 45(3)

Douthwaite M, Ward P. Increasing contraceptive use in rural Pakistan: an evaluation of the Lady Health Worker Programme. Health Policy Plan. 2005; 20(2):117-123. [PubMed: 15746220]

Eggleston E, Jackson J, Rountree W, Pan Z. Evaluation of a sexuality education program for young adolescents in Jamaica. Rev Panam Salud Publica. 2000; 7(2):102-112. [PubMed: 10748661]

Ensor T, Cooper S. Overcoming barriers to health service access: influencing the demand side. Health Policy and Planning. 2004; 19(2):69-79. [PubMed: 14982885]

Erulkar AS, Ettyang L, Onoka C, Nyagah C, Muyonga A. Behavior change evaluation of a culturally consistent reproductive health program for young Kenyans. International Family Planning Perspectives. 2004; 30(2):58-67. [PubMed: 15210404]

FOCUS on Young Adults and CARE International-Cambodia. Impact of an Adolescent Reproductive Health Education Intervention Undertaken in Garment Factories in Phnom Penh, Cambodia. Washington, DC: 2000.

Glasier A, Gülmezoglu A, Schmid G, Moreno C, Van Look P. Sexual and reproductive health: a matter of life and death. The Lancet. 2006; 368(9547):1595-1607.

Global HIV Prevention Working Group. Behavior Change and HIV Prevention: [Re]Considerations for the 21stCentury. 2008. www.GlobalHIVPrevention.org

Gupta G, Parkhurst J, Ogden J, Aggleton P, Mahal A. Structural Approaches to HIV Prevention. The Lancet. 2008a; 372:764-775.

Hennink M, Clements S. The impact of franchised family planning clinics in poor urban areas of Pakistan. Stud Fam Plann. 2005; 36(1):33-44. [PubMed: 15828523]

Hermalin A. Family planning impact evaluation: the evolution of techniques. Población y Salud en Mesoamérica. 2003; 1(1):1-37.

Islam, M. Vouchers for health: a focus on reproductive health and family planning services. Bethesda, Maryland: Abt Associates, Private Sector Partnerships One [PSP-One]; 2006.

Khan, ME.; Hossain, S.; Rahman, M. Introduction of Emergency Contraception in Bangladesh: Using Operations Research for Policy Decisions. Washington, DC: FRONTIER, Population Council; 2004.

Khan, ME.; Phillip Sebastian, M.; Sharma, U.; Idnani, R.; Kumari, K.; Maheshwari, B., et al. Promoting Healthy Timing and Spacing of Births in India through a Community-based Approach. Washington, DC: FRONTIER, Population Council; 2008.

Kim YM, Kols A, Nyakauru R, Marangwanda C, Chibatamoto P. Promoting sexual responsibility among young people in Zimbabwe. International Family Planning Perspectives. 2001 Mar; 27(1): $11-19$. 
Kincaid DL. Mass media, ideation, and behavior: a longitudinal analysis of contraceptive change in the Philippines. Communication Research. 2000a; 27(6):723-763.

Kincaid DL. Social networks, ideation, and contraceptive behavior in Bangladesh: a longitudinal analysis. Social Science \& Medicine. 2000b; 50(2):215-231. [PubMed: 10619691]

Kunene, B.; Beksinska, M.; Zondi, S.; Mthembu, N.; Mullick, S.; Ottolenghi, E., et al. Involving Men in Maternity Care, South Africa. Washington, DC: FRONTIER, Population Council; 2004.

Lapham RJ, Mauldin WP. Contraceptive Prevalence: The Influence of Organized Family Planning Programs. Studies in Family Planning. 1985; 16(3):117-137. [PubMed: 4012818]

LaVake, SD. Applying Social Franchising Techniques to Youth Reproductive Health/HIV Services. Arlington, VA: Family Health International, YouthNet Program; 2003.

Levitt-Dayal, M.; Motihar, R. Adolescent Girls in India Choose a Better Future: An Impact Assessment. CEDPA; India: 2001.

Lloyd, C.; Ross, J. Research Division Working Papers No 7. NY: The Population Council; 1989. Methods for measuring the fertility impact of family planning programs: The experience of the last decade.

Lou CH, Wang B, Shen Y, Gao ES. Effects of a community-based sex education and reproductive health service program on contraceptive use of unmarried youths in Shanghai. Journal of Adolescent Health. 2004; 34(5):433-440. [PubMed: 15093800]

Magnani, R.; Robinson, A.; Seiber, E. Evaluation of 'Arte y Parte': An Adolescent Reproductive Health Communications Project Implemented in Asunción, San Lorenzo and Fernando de la Mora, Paraguay. Washington, DC: FOCUS on Young Adults Program; 2000a.

Magnani, R.; Gaffikin, L.; Espinoza, V.; Zielinski Gutierrez, E.; Rivas, MI.; Seiber, E., et al. Evaluation of Juventud EsSalud: An Adolescent Reproductive and Sexual Health Peer Education Program Implemented in Six Departments in Peru. Tulane University School of Public Health and Tropical Medicine/FOCUS on Young Adults Program; 2000b Jul.

Magnani R. Impact of an integrated adolescent reproductive health program in Brazil. Studies in Family Planning. 2001; 32(3):230-243. [PubMed: 11686184]

Martiniuk ALC, O'Connor KS, King WD. A cluster randomized trial of a sex education programme in Belize, Central America. Int J Epidemiol. 2003; 32(1):131-136. [PubMed: 12690024]

Mathur, MM.; A, M. Youth reproductive health in Nepal: Is participation the answer?. Washington, DC: International Center for Research on Women [ICRW]; 2004 Jan.

Mba C, Obi SN, Ozumba BC. The impact of health education on reproductive health knowledge among adolescents in a rural Nigerian community. Journal of Obstetrics and Gynaecology. 27(5): 513-517. [PubMed: 17701804]

Mbizvo MT, Kasule J, Gupta V, Rusakaniko S, Kinoti SN, Mpanju-Shumbushu W, et al. Effects of a randomized health education intervention on aspects of peproductive health knowledge and reported behaviour among adolescents in Zimbabwe. Social Science \& Medicine. 1997; 44(5): 573-577. [PubMed: 9032825]

Meekers, D.; Stallworthy, G.; H, J. Working Paper No 3. Washington, DC: Population Services International [PSI], Research Division; 1997. Changing adolescents' beliefs about protective sexual behavior: the Botswana Tsa Banana program.

Meekers, D. Working Paper No 16. Washington, DC: Population Services International [PSI], Research Division; 1998. The Effectiveness of Targeted Social Marketing to Promote Adolescent Reproductive Health: The Case of Soweto, South Africa.

Meuwissen LE, Gorter AC, Knottnerus AJA. Impact of accessible sexual and reproductive health care on poor and underserved adolescents in Managua, Nicaragua: a quasi-experimental intervention study. Journal of Adolescent Health. 2006; 38(1):56.e1-56.e9. [PubMed: 16387251]

Moreland, S.; Talbird, S. Achieving the Millennium Development Goals: The contribution of fulfilling the unmet need for family planning. Washington, DC: Futures Group/POLICY Project; May. 2006

Murray NT, Luengo X, Molina R, Zabin L. An evaluation of an integrated adolescent development program for urban teenagers in Santiago, Chile. 2000

Nag, M. No 1041, Policy Research Working Paper Series. Washington, DC: The World Bank; 1992. Family planning success stories in Bangladesh and India. 
Nawar, L.; Kharboush, I.; Ibrahim, MA.; Makhlouf, H.; Adamchak, S. Impact of Improved ClientProvider Interaction on Women's Achievement of Fertility Goals in Egypt. Washington, DC: FRONTIER, Population Council; 2004.

Özcebe H, Akin L. Effects of peer education on reproductive health knowledge for adolescents living in rural areas of Turkey. Journal of Adolescent Health. 2003; 33(4):217-218. [PubMed: 14519561]

Phillips JF, Hossain MB, Arends-Kuenning M. The long-term demographic role of community-based family planning in rural Bangladesh. Stud Fam Plann. 1996; 27(4):204-219. [PubMed: 8875733]

Population Reference Bureau [PRB]. Family Planning Worldwide 2008 Data Sheet. Washington, DC: PRB; 2008.

Potts M, Fotso J. Population growth and the Millennium Development Goals. The Lancet. 2007; 369(9559):354-355.

Rahman M, DaVanzo J, Razzaque A. Do better family planning services reduce abortion in Bangladesh? Lancet. 2001; 358(9287):1051-1056. [PubMed: 11589936]

Richey, C.; Salem, RM. Elements of Success in Family Planning Programming. Baltimore, MD: INFO Project, Johns Hopkins Bloomberg School of Public Health; Sep. 2008

Rogers EM, Vaughan PW, Swalehe RMA, Rao N, Svenkerud P, Sood S. Effects of an entertainmenteducation radio soap opera on family planning behavior in Tanzania. Studies in Family Planning. 1999; 30(3):193-211. [PubMed: 10546311]

Ross DA, Changalucha J, Obasi AI, Todd J, Plummer ML, Cleophas-Mazige B, et al. Biological and behavioural impact of an adolescent sexual health intervention in Tanzania: a communityrandomized trial. AIDS. 2007; 21(14):1943-1955. [PubMed: 17721102]

Rusakaniko S, Mbizvo MT, Kasule J, Gupta V, Kinoti SN, Mpanju-Shumbushu W, et al. Trends in reproductive health knowledge following a health education intervention among adolescents in Zimbabwe. Cent Afr J Med. 1997; 43(1):1-6. [PubMed: 9185371]

Salem, RM.; Bernstein, J.; Sullivan, TM.; Lande, R. Communication for Better Health. Baltimore, MD: INFO Project, Johns Hopkins Bloomberg School of Public Health; Jan. 2008

Samara, R.; Buckner, B.; Tsui, AO. Understanding how family planning programs work: Findings from five years of evaluation research. Chapel Hill, NC: The EVALUATION Project; 1996.

Sanogo D, RamaRao S, Jones H, N'diaye P, M'bou B, Diop CB. Improving quality of care and use of contraceptives in Senegal. Rev Afr Santé Reprod. 2003; 7(2):57-73.

Shelton J, et al. Putting unmet need to the test: community-based distribution of family planning in Pakistan. International Family Planning Perspectives. 1999; 25(4):191-195.

Sherwood-Fabre L, Goldberg H, Bodrova V. The impact of an integrated family planning program in Russia. Eval Rev. 2002; 26(2):190-212. [PubMed: 11949538]

Shuey DA, Babishangire BB, Omiat S, Bagarukayo H. Increased sexual abstinence among in-school adolescents as a result of school health education in Soroti district, Uganda. Health Educ Res. 1999; 14(3):411-419. [PubMed: 10539231]

Signorini, Queiroz. The impact of Bolsa Familia program in the beneficiary fertility. 2009 draft.

Sinding, SW. Overview and Perspective. In: Robinson, Warren C.; Ross, John A., editors. The Global Family Planning Revolution: Three Decades of Population Policies and Programs. Washington, DC: The International Bank for Reconstruction and Development / The World Bank; 2007.

Sinha N. Fertility, child work, and schooling consequences of family planning programs: Evidence from an experiment in rural Bangladesh. Economic Development and Cultural Change. 2005; 54(1):97-128.

Sood S, Sengupta M, Mishra PR, Jacoby C. 'Come gather around together': an examination of radio listening groups in Fulbari, Nepal. Gazette. 2004; 66(1):63-86.

Speizer IS, Tambashe BO, Tegang SP. An evaluation of the 'Entre Nous Jeunes' peer-educator program for adolescents in Cameroon. Studies in Family Planning. 2001; 32(4):339-351. [PubMed: 11831052]

Speizer IS, Magnani RJ, Colvin CE. The effectiveness of adolescent reproductive health interventions in developing countries: a review of the evidence. Journal of Adolescent Health. 2003; 33(5):324348. [PubMed: 14596955] 
Speizer IS, Kouwonou K, Mullen S, Vignikin E. Evaluation of the ATBEF Youth Centre in Lome, Togo. African Journal of Reproductive Health. 2004; 8(3):38-54. [PubMed: 17348324]

Stanton B, Li X, et al. Increased protected sex and abstinence among Namibian youth following a HIV risk-reduction intervention: a randomized, longitudinal study. AIDS. 12(18):2473-80. [PubMed: 9875586]

Stecklov G, Winters P, Todd J, Regalia F. Unintended effects of poverty programmes on childbearing in less developed countries: experimental evidence from Latin America. Popul Stud (Camb). 2007; 61(2):125-140. [PubMed: 17558882]

Steele F, Amin S, Naved RT. Savings/credit group formation and change in contraception. Demography. 2001; 38(2):267-282. [PubMed: 11392912]

Tu X, Lou C, Gao E, Shah IH. Long-term effects of a community-based program on contraceptive use among sexually active unmarried youth in Shanghai, China. Journal of Adolescent Health. 2008; 42(3):249-258. [PubMed: 18295133]

United Nations. Manual IX: The Methodology of Measuring the Impact of Family Planning Programs on Fertility, Addendum. New York: UN; 1986.

United Nations. Manual IX: The Methodology of Measuring the Impact of Family Planning Programs on Fertility. New York: UN; 1979.

United Nations Population Fund. State of the world population 2005. New York: UN Population Fund; 2005.

Van Rossem, R.; Meekers, D. Working Paper No 19. Washington, DC: PSI, Research Division; 1999a. An Evaluation of the Effectiveness of Targeted Social Marketing to Promote Adolescent and Young Adult Reproductive Health in Cameroon.

Van Rossem, R.; Meekers, D. Working Paper No 23. Washington, DC: PSI, Research Division; 1999b. An Evaluation of the Effectiveness of Targeted Social Marketing to Promote Adolescent Reproductive Health in Guinea.

Varkey, C.; Mishra; Das; Ottolenghi; Huntington; Adamchak, et al. Involving Men in Maternity Care in India. Washington, DC: FRONTIER, Population Council; 2004.

Vernon, R.; Dura, M. Improving the Reproductive Health of Youth In Mexico. Washington, DC: FRONTIER, Population Council; 2004.

Victora CG, Habicht J-P, Bryce J. Evidence-based public health: moving beyond randomized trials. American Journal of Public Health. 2004; 94(3):400-05. [PubMed: 14998803]

Walker D, Gutierrez JP, Torres P, Bertozzi SM. HIV prevention in Mexican schools: prospective randomized evaluation of intervention. BMJ. 2006:1-6.

William, D.; Savedoff, WD.; Levine, R.; Birdsal, N. Report of the Evaluation Gap Working Group. Washington, DC: Center for Global Development; 2006. When Will We Ever Learn? Improving Lives through Impact Evaluation.

World Health Organization. Global and regional estimates of the incidence of unsafe abortion and associated mortality in 2000. Geneva: WHO; 2004.

Xiaoming S, Yong W, Choi K-H, Lurie P, Mandel J. Integrating HIV Prevention Education into Existing Family Planning Services: Results of a Controlled Trial of a Community-Level Intervention for Young Adults in Rural China. AIDS and Behavior. 2000; 4(1):103-110. 
Table 1

Strength of Evidence of Included Family Planning Evaluation Studies by Type of Intervention and Strength of Evidence

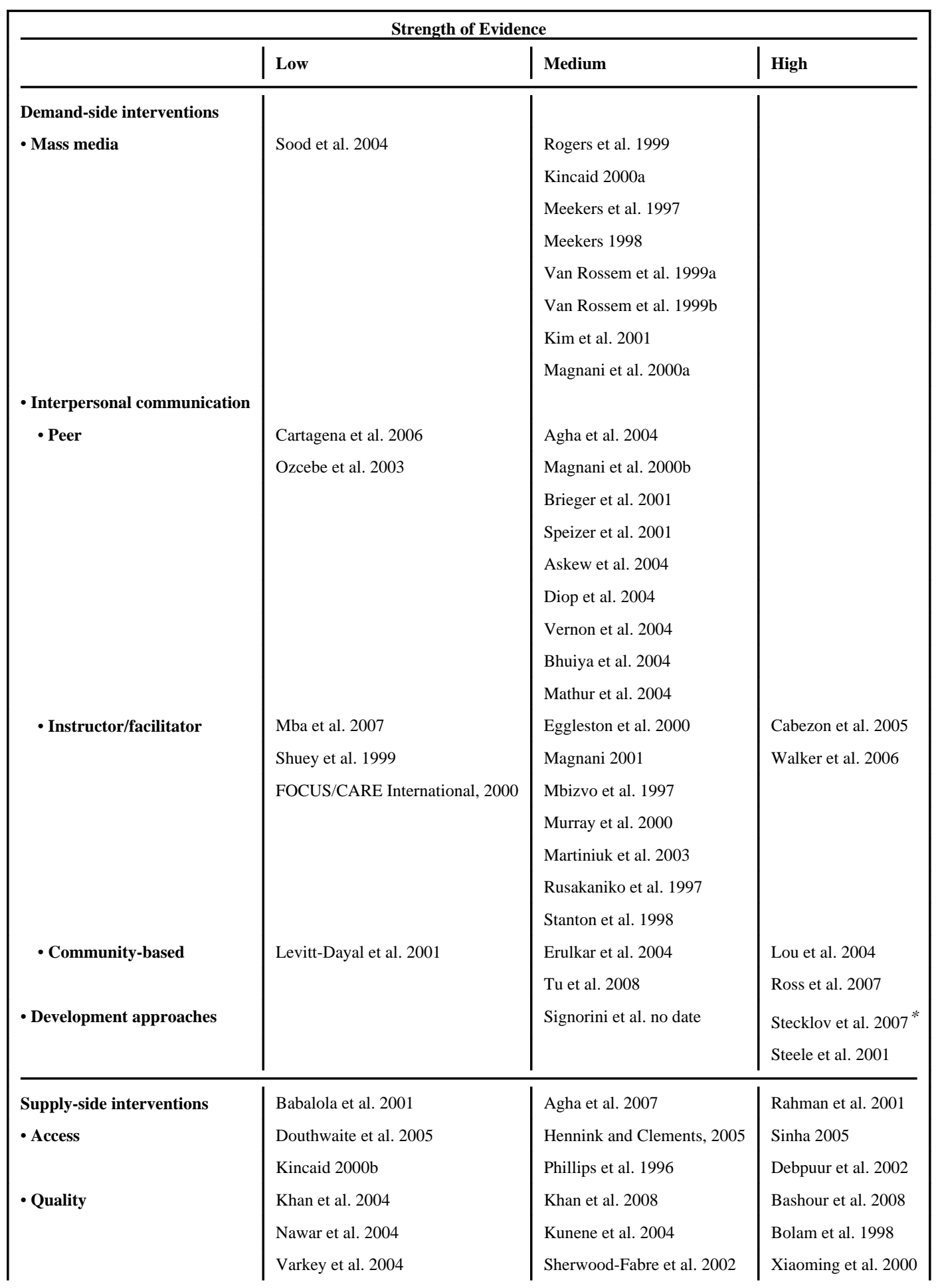




\begin{tabular}{|l|l|l|l|}
\hline & \multicolumn{4}{c|}{ Strength of Evidence } & High \\
\hline & Low & Medium & \\
\hline Cost & Speizer et al. 2004 & Sanogo et al. 2003 & \\
\hline
\end{tabular}

Included as 3 separate studies 


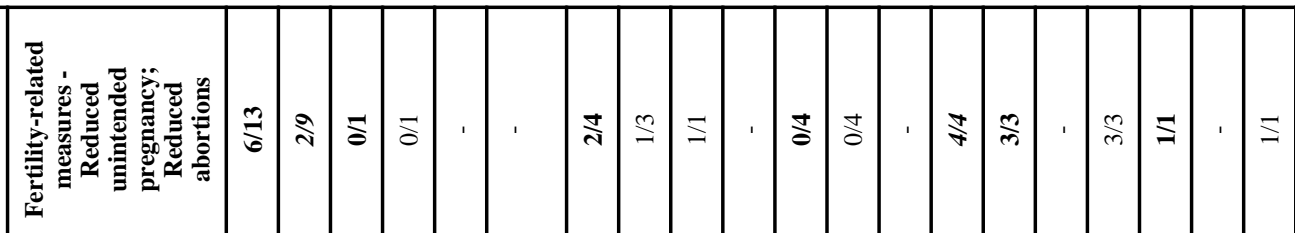

苛苛

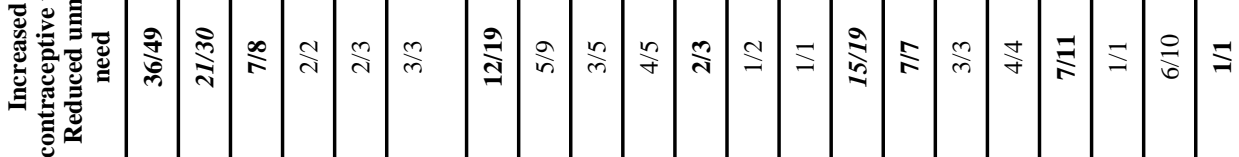




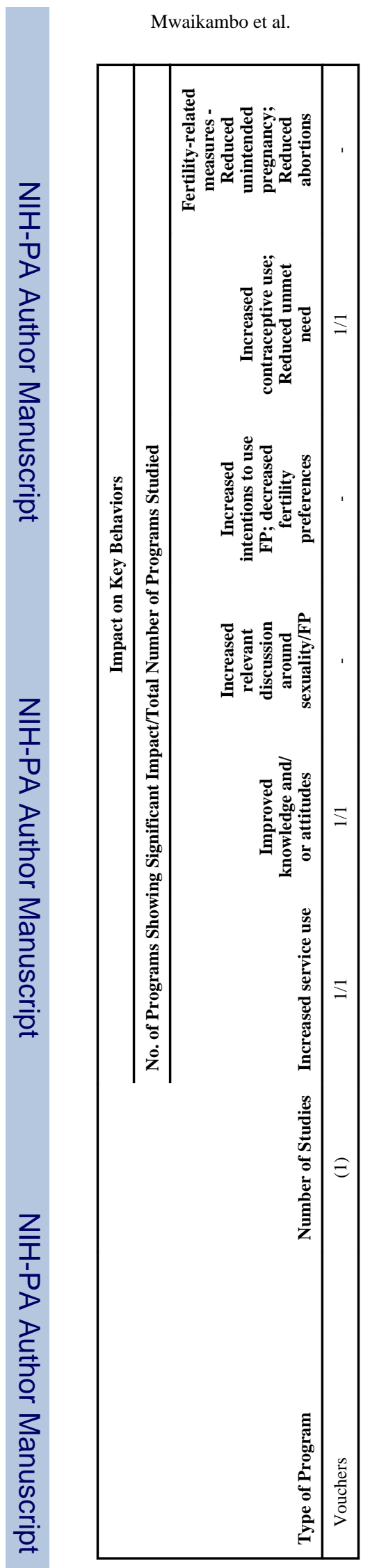

Stud Fam Plann. Author manuscript; available in PMC 2013 September 03. 


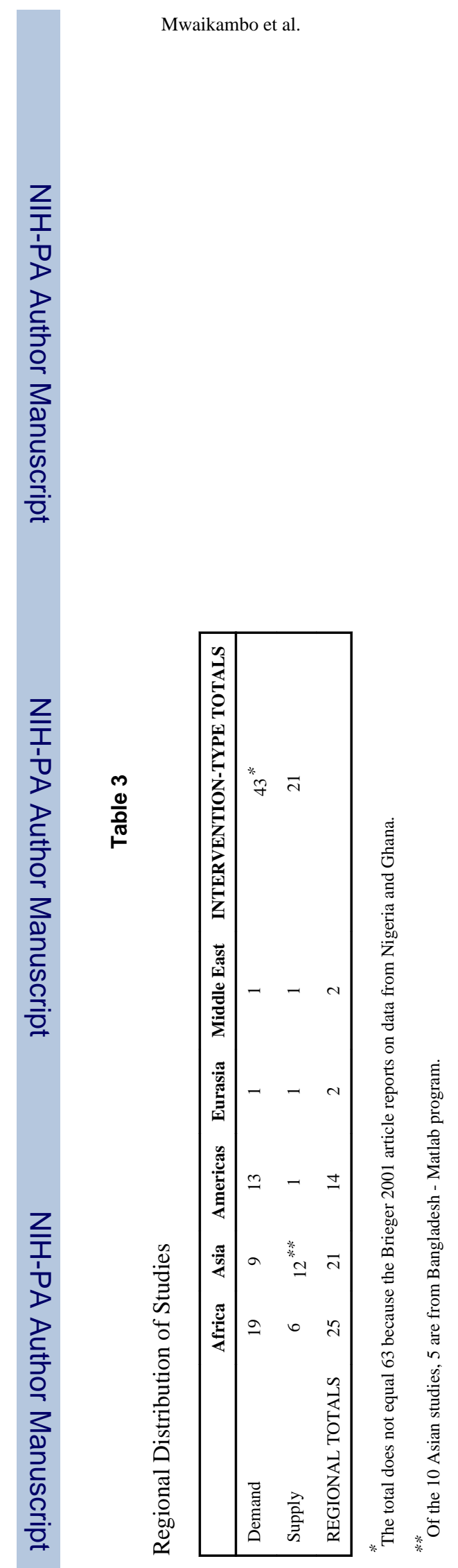

Stud Fam Plann. Author manuscript; available in PMC 2013 September 03. 


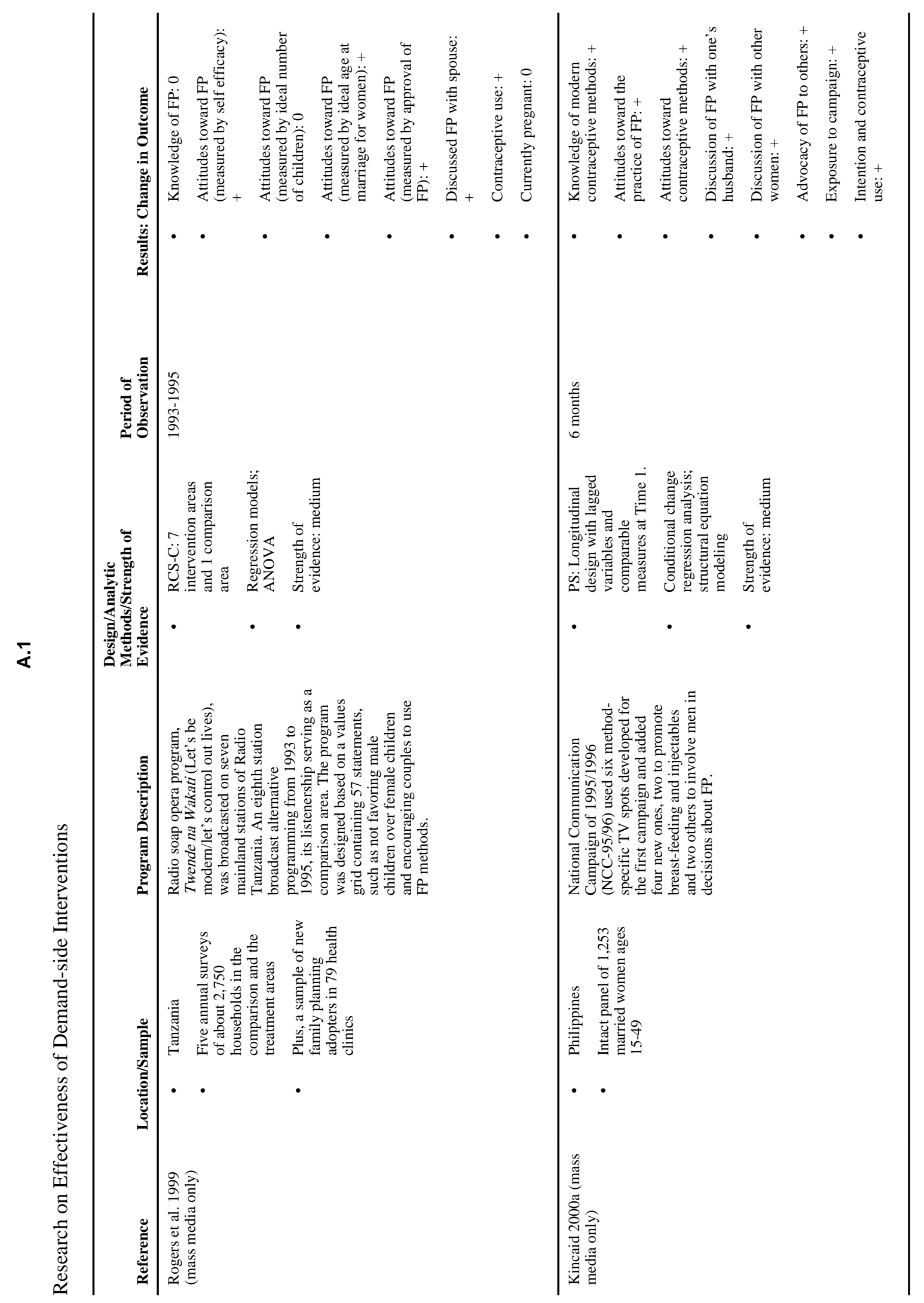


Mwaikambo et al.

Page 29

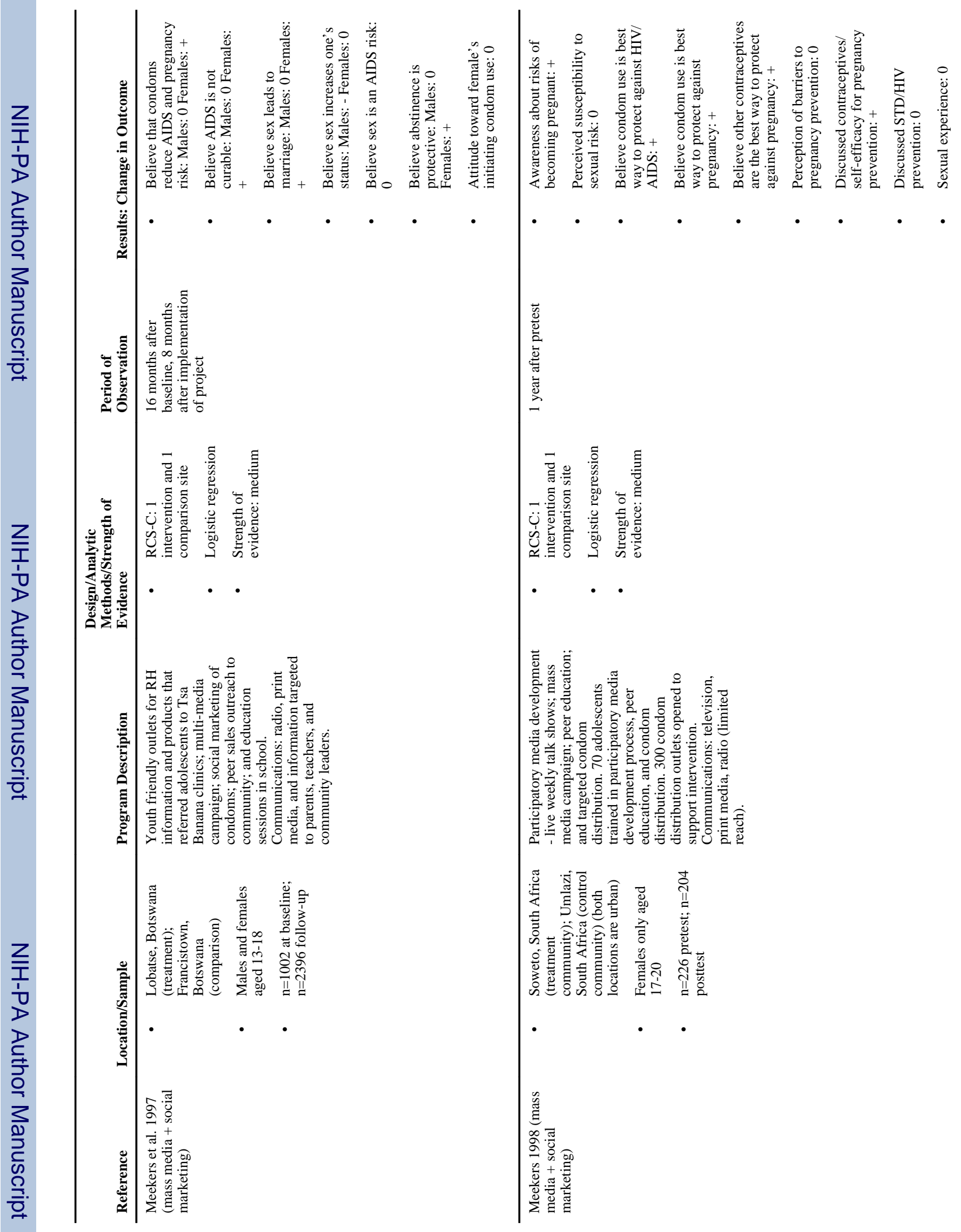


Mwaikambo et al.

Page 30

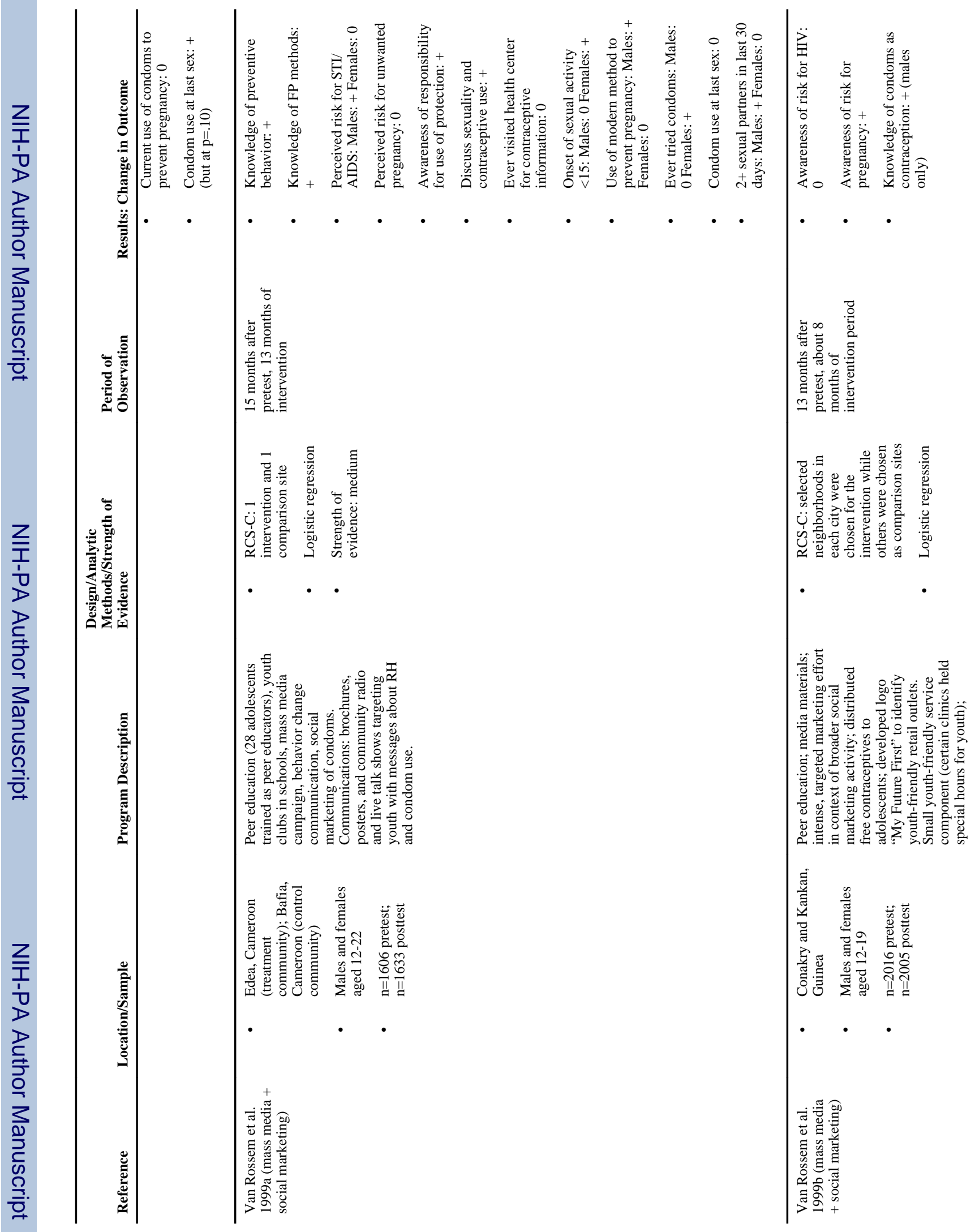

Stud Fam Plann. Author manuscript; available in PMC 2013 September 03. 


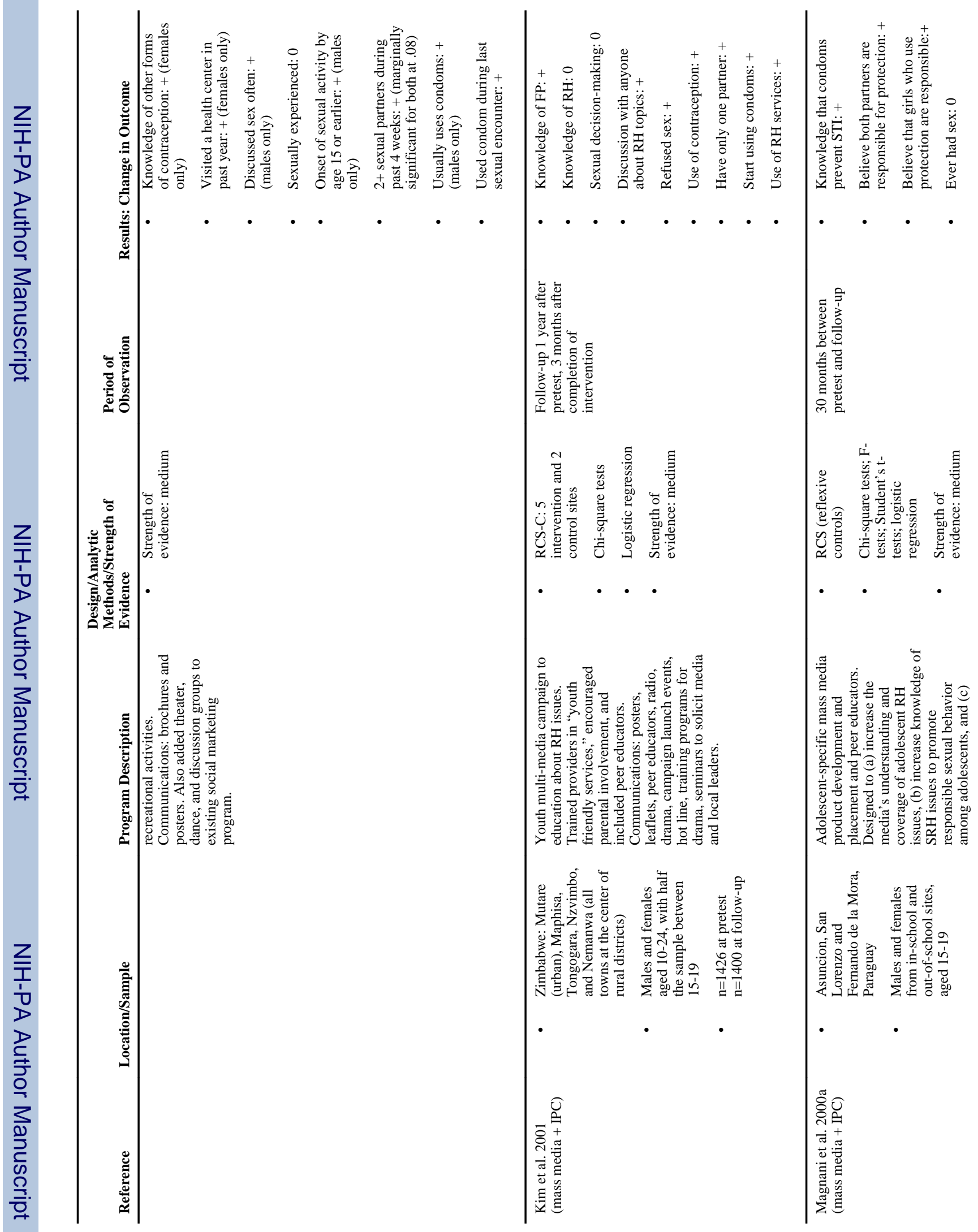




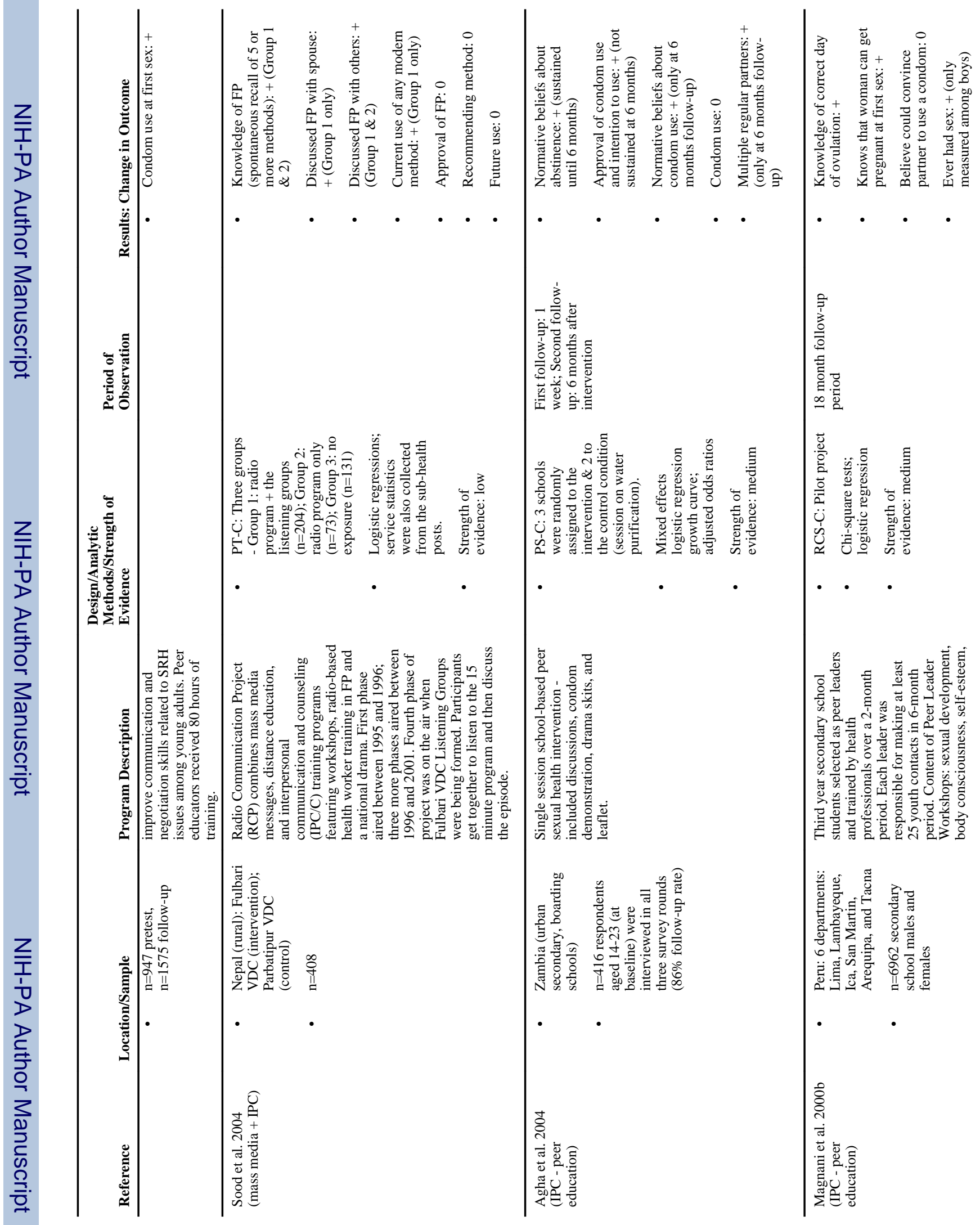




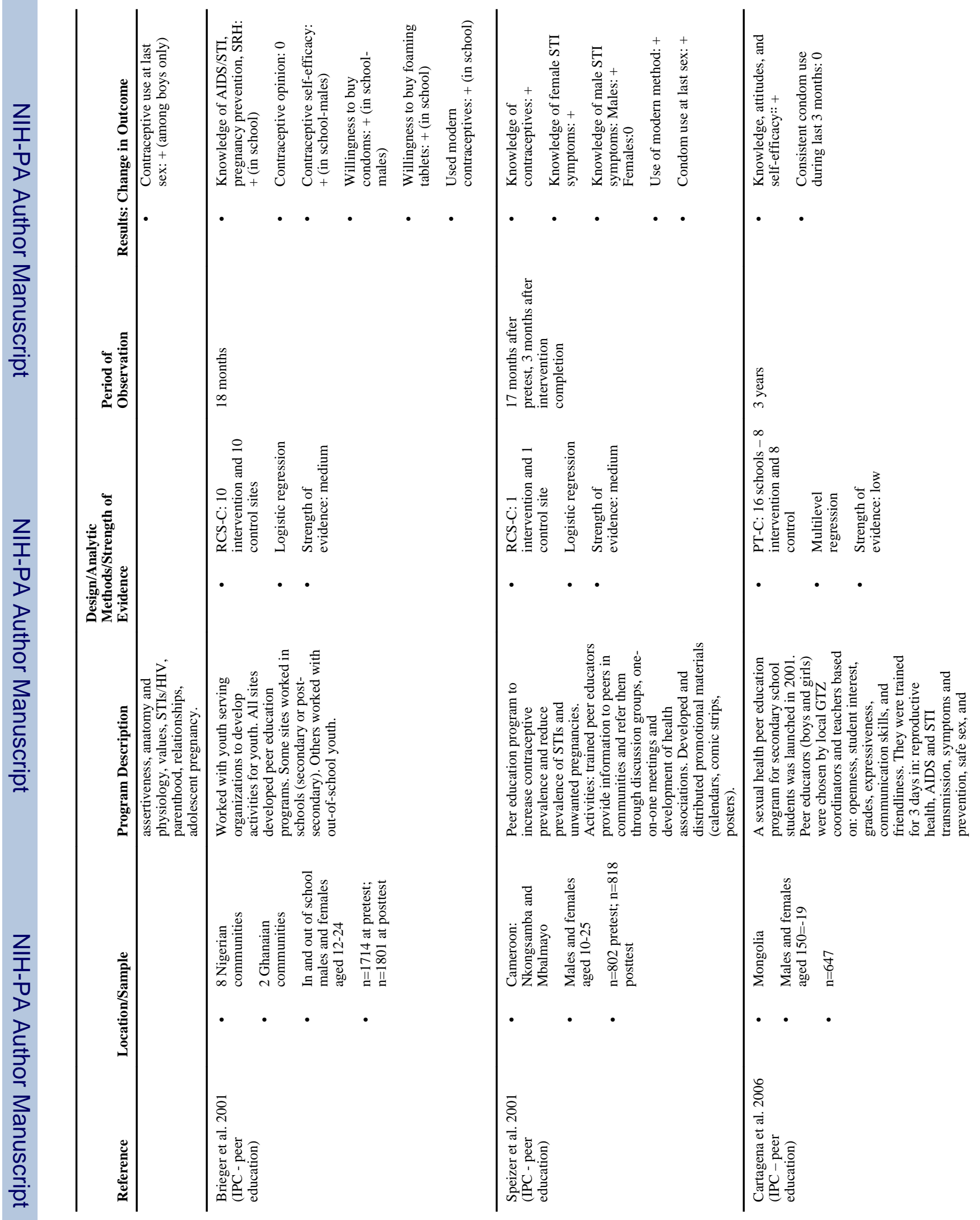




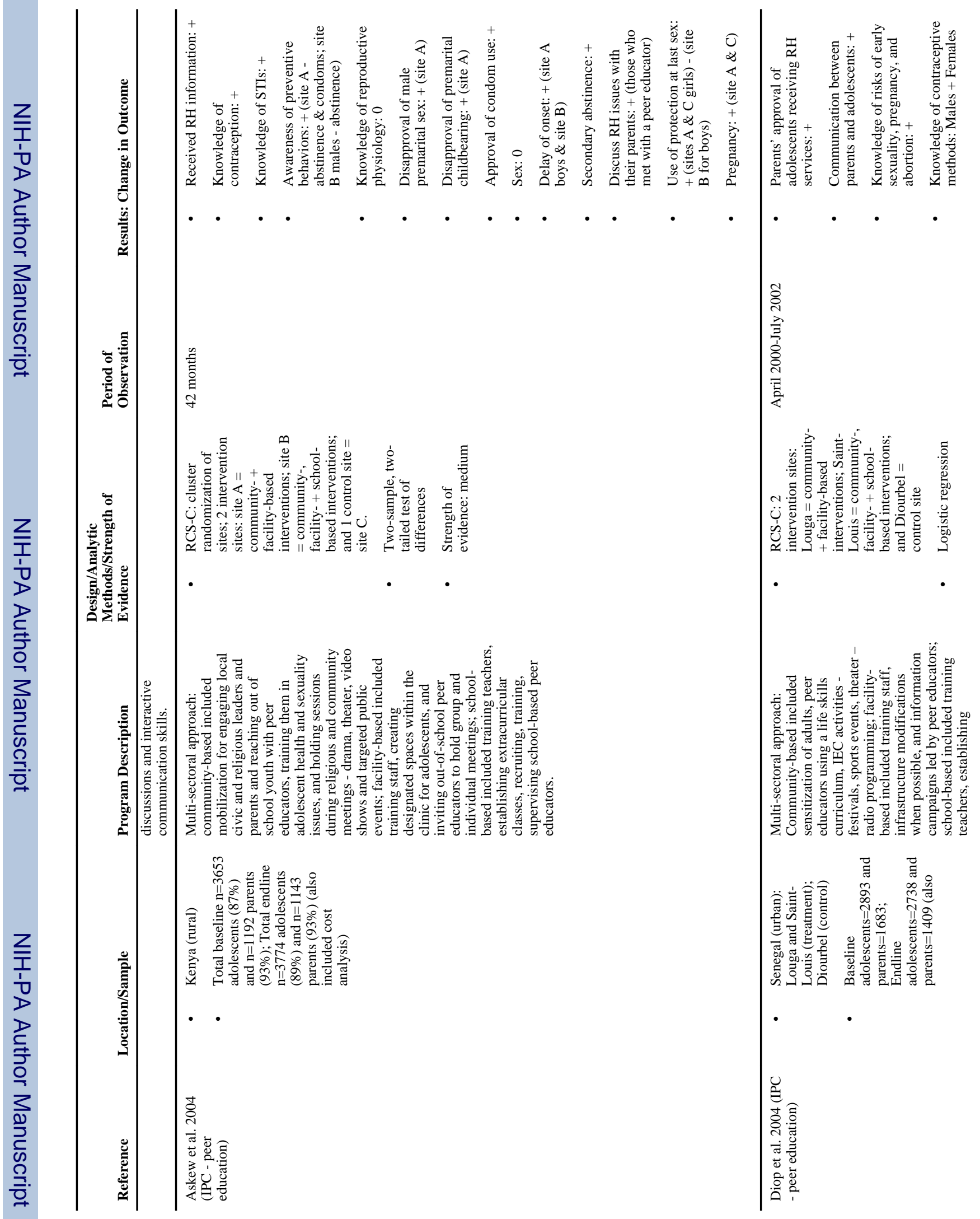




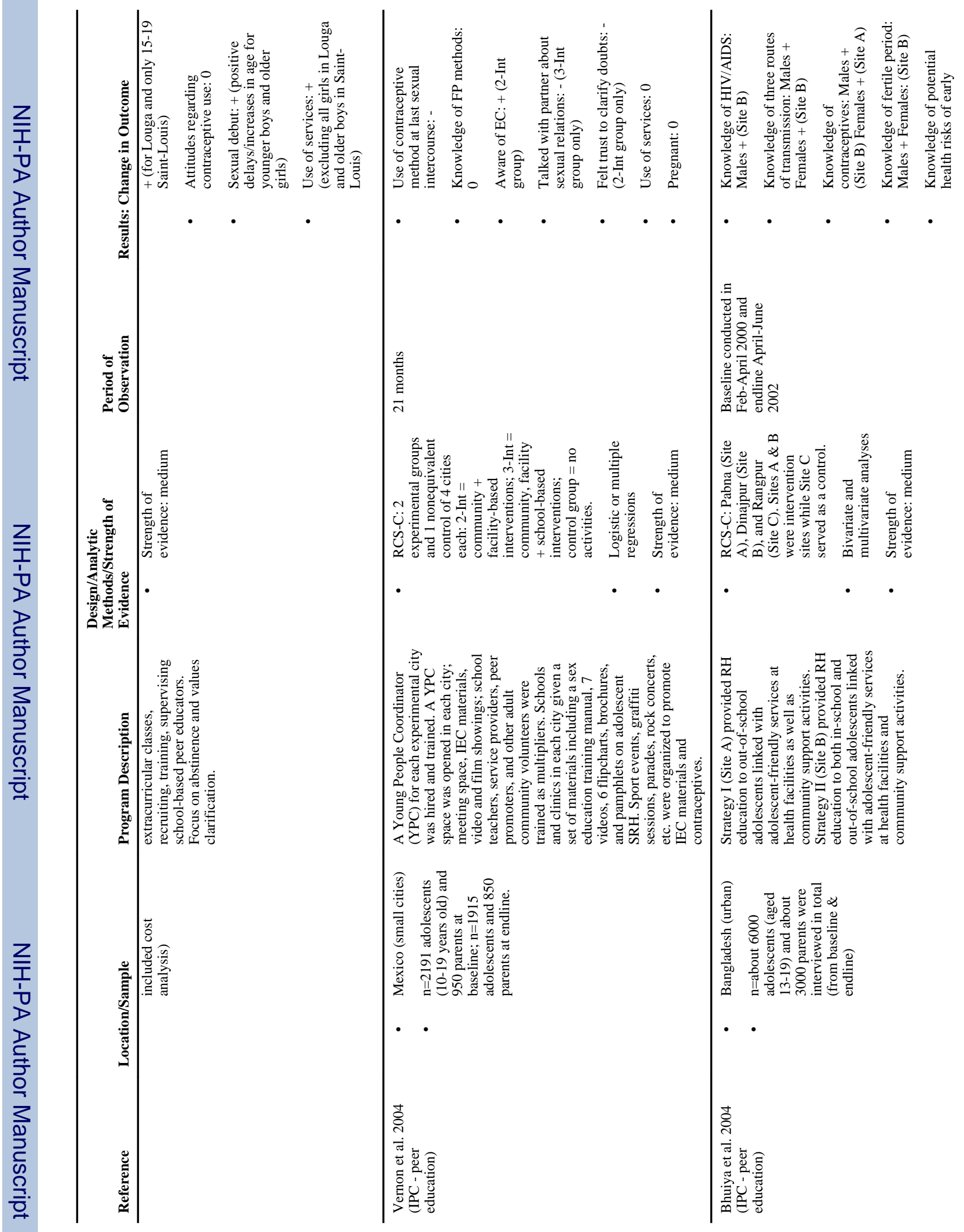




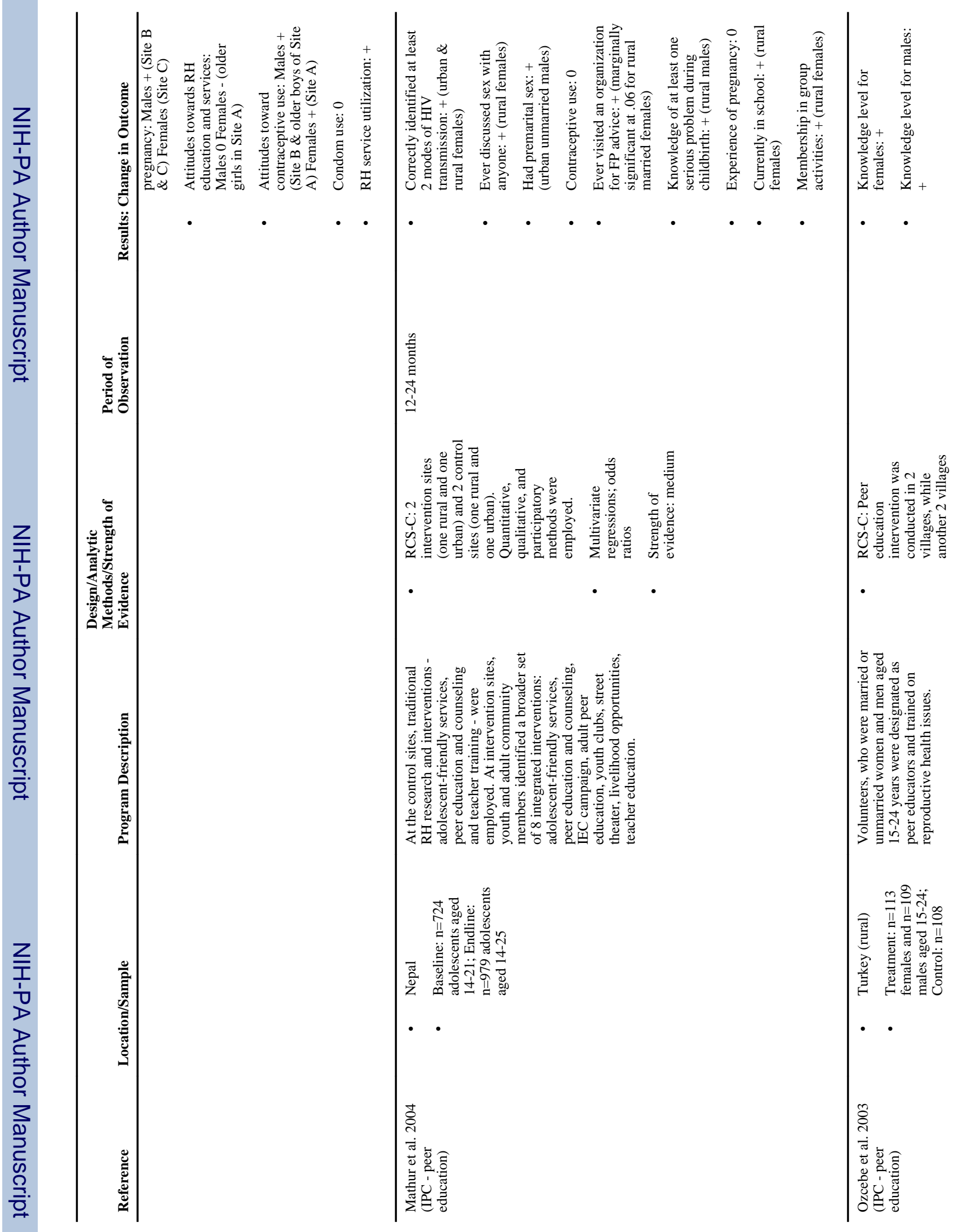




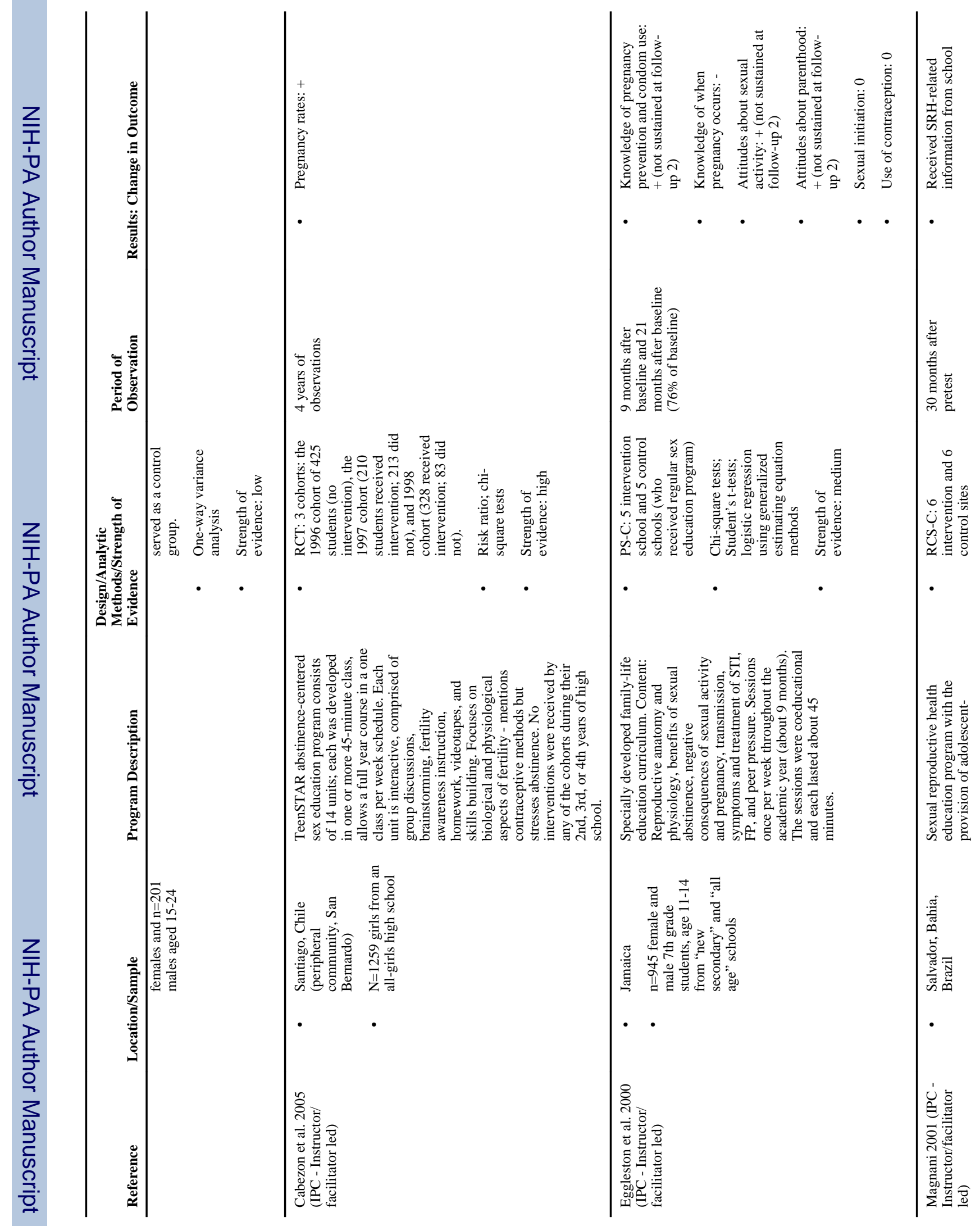




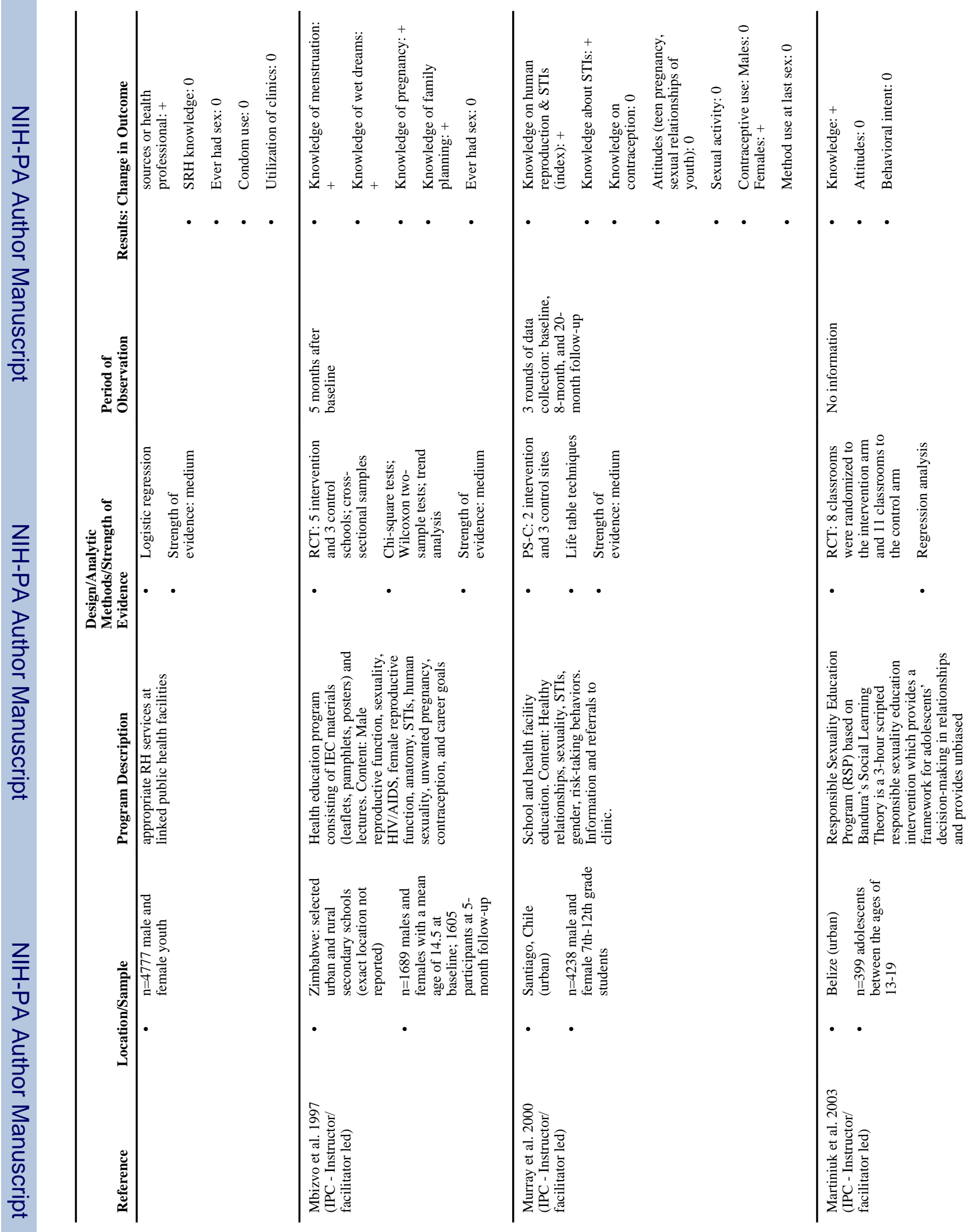


Mwaikambo et al.

Page 39

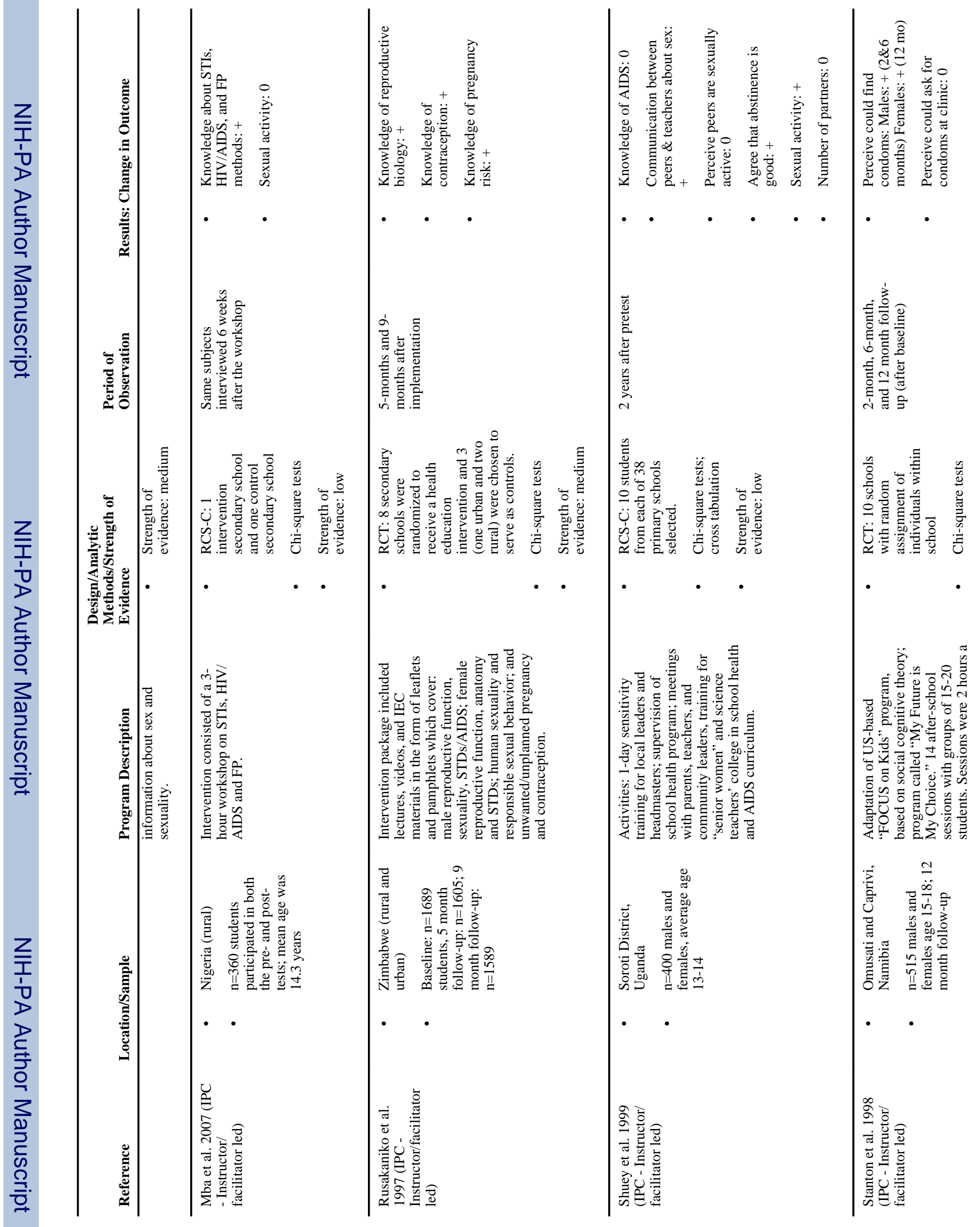

Stud Fam Plann. Author manuscript; available in PMC 2013 September 03. 


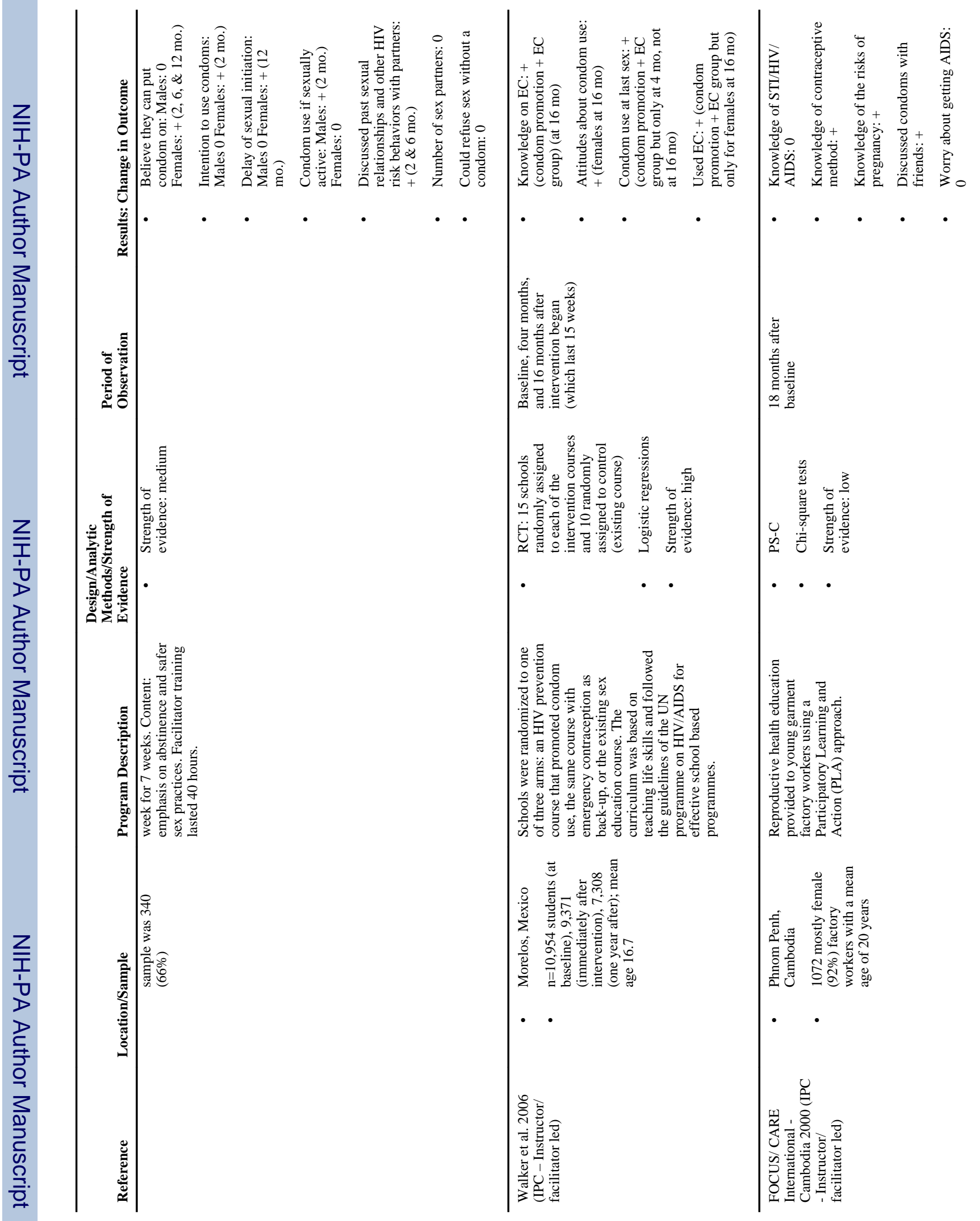


Mwaikambo et al.

Page 41

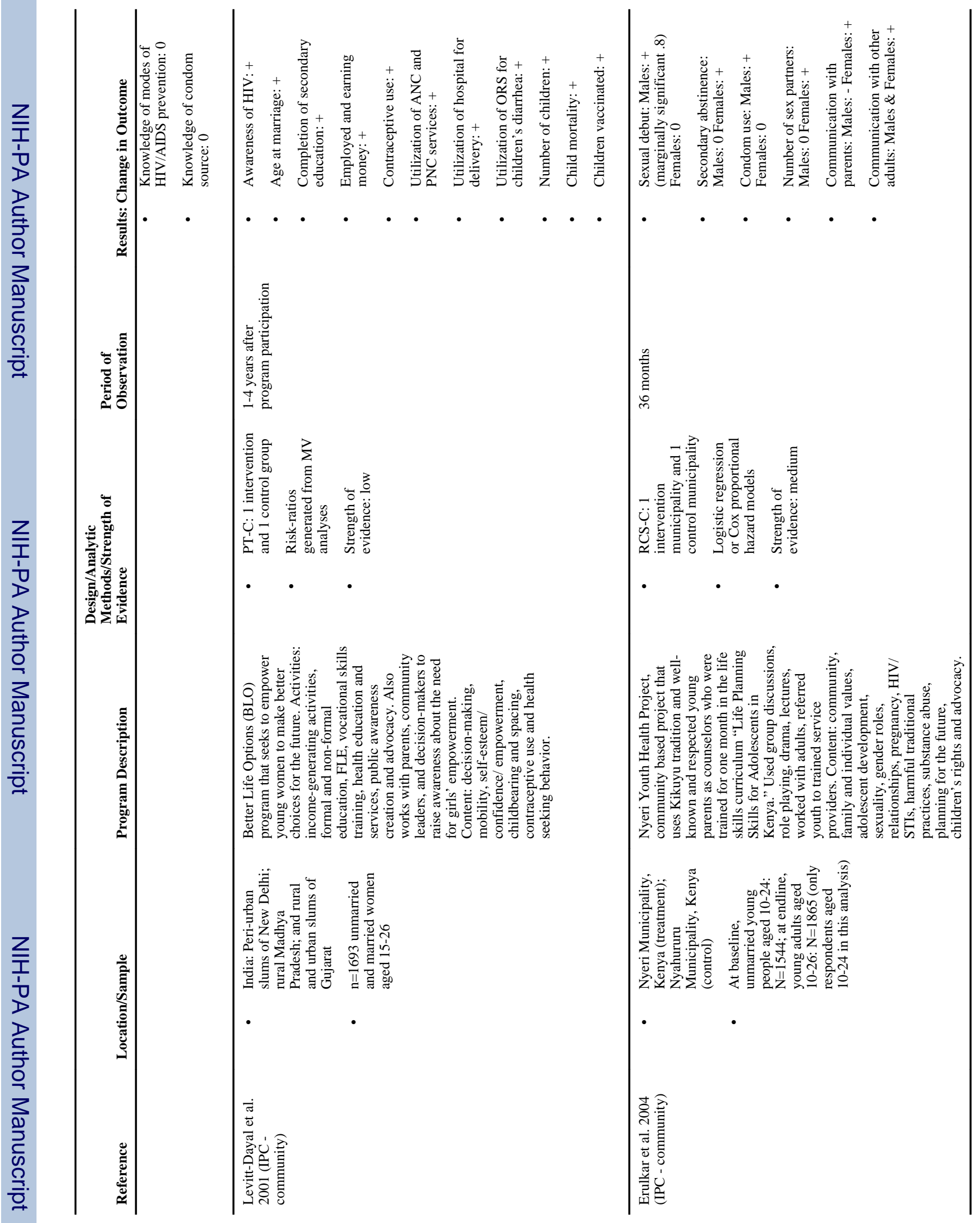




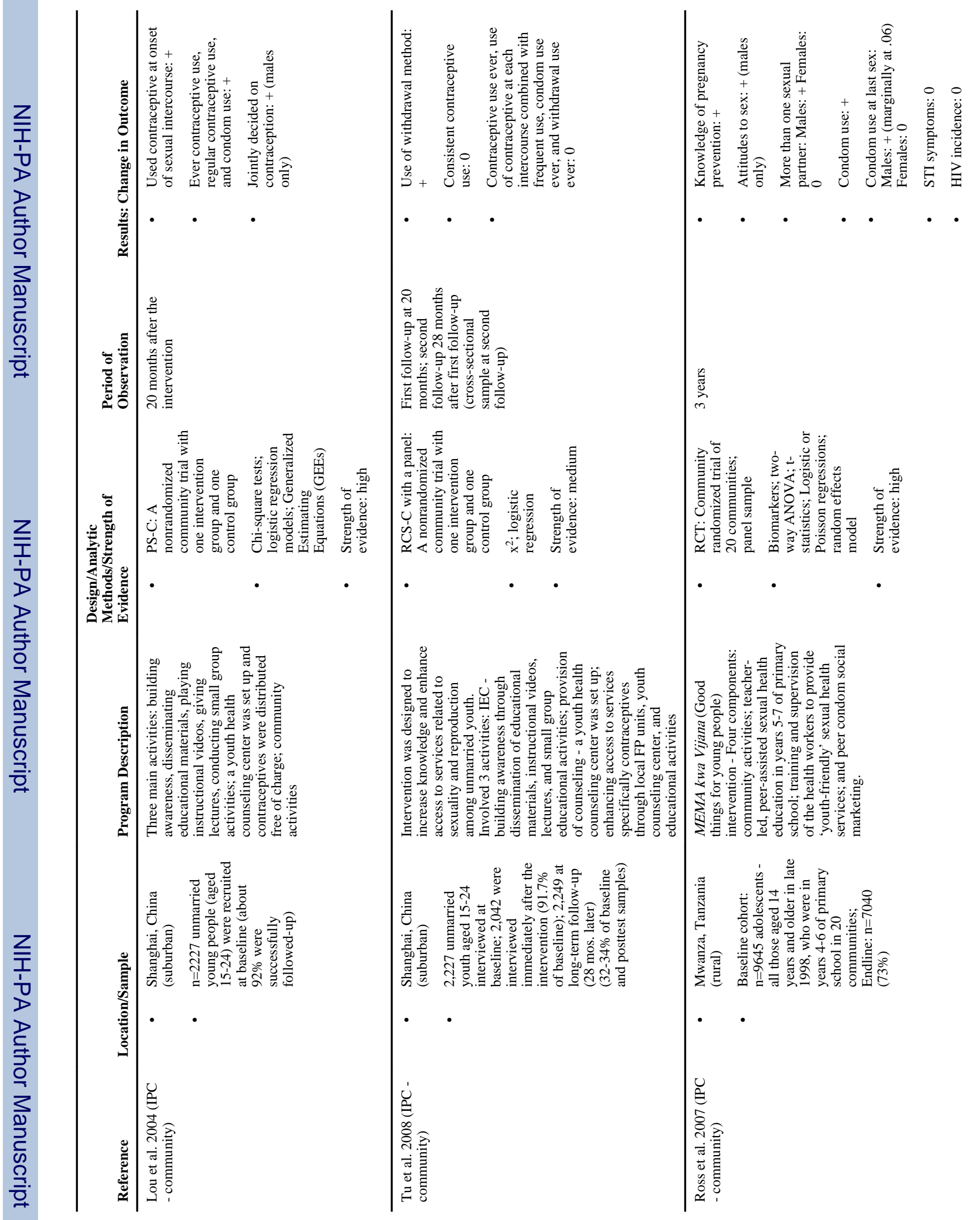




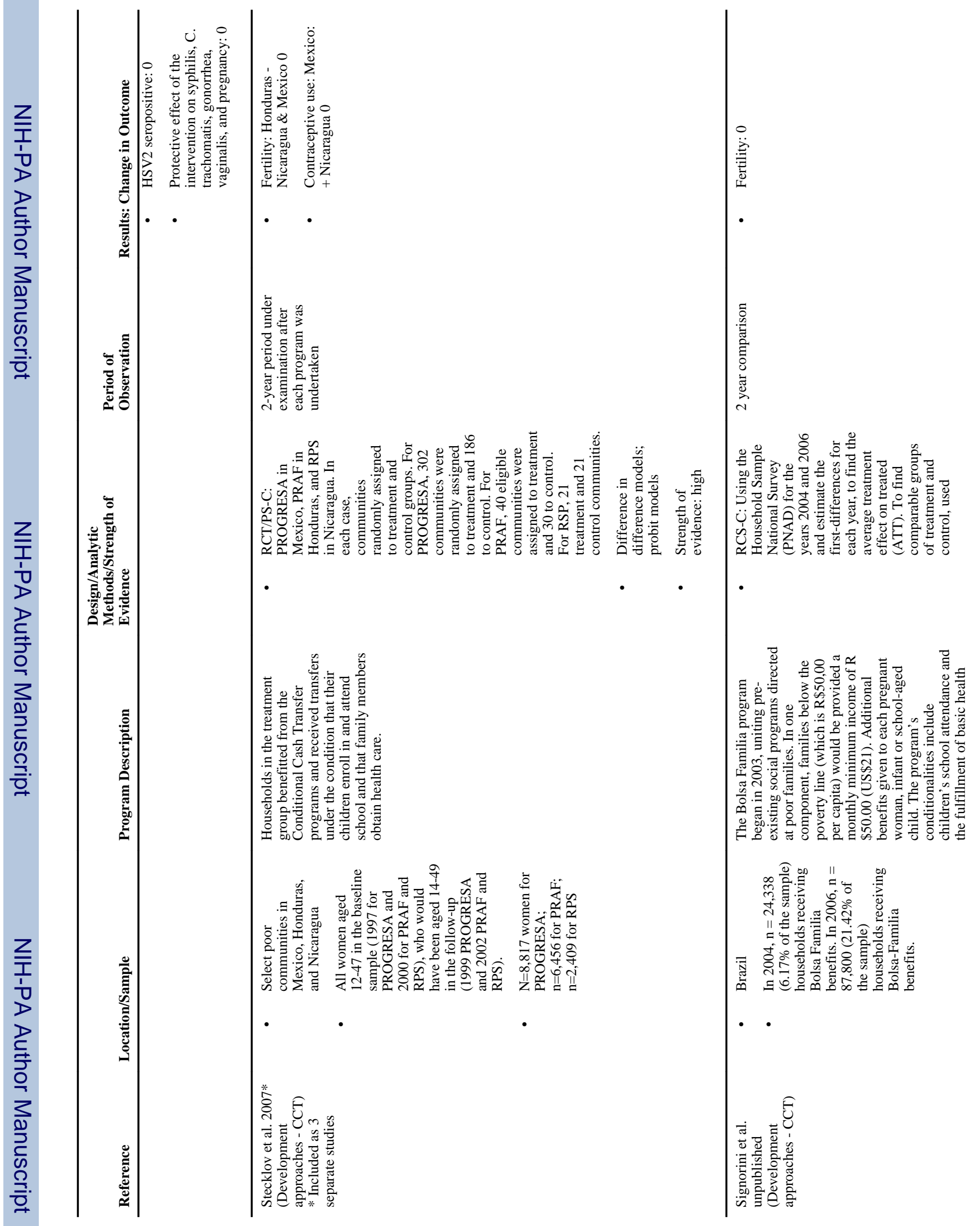



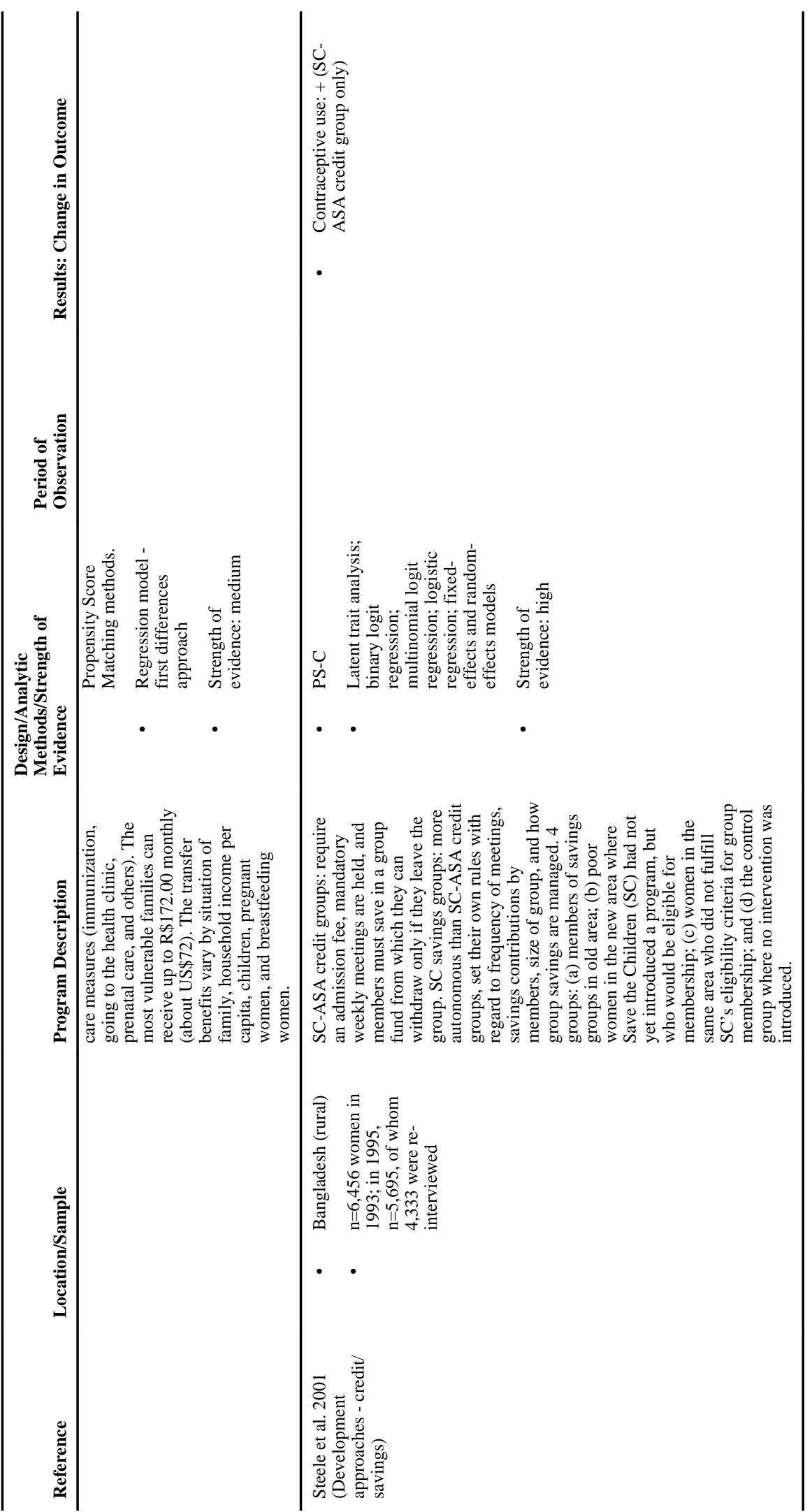

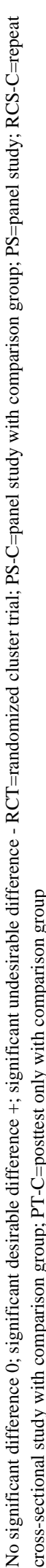




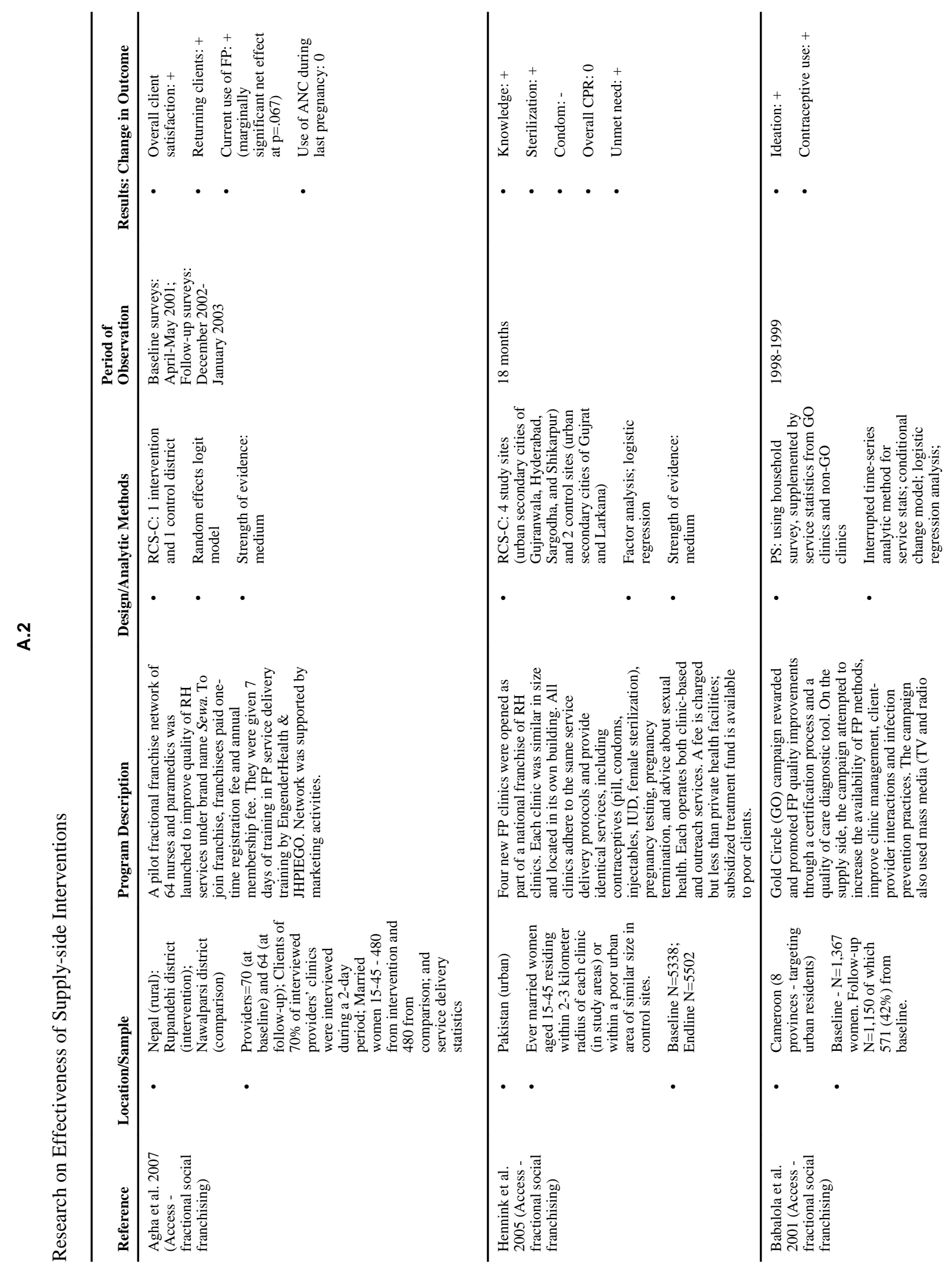




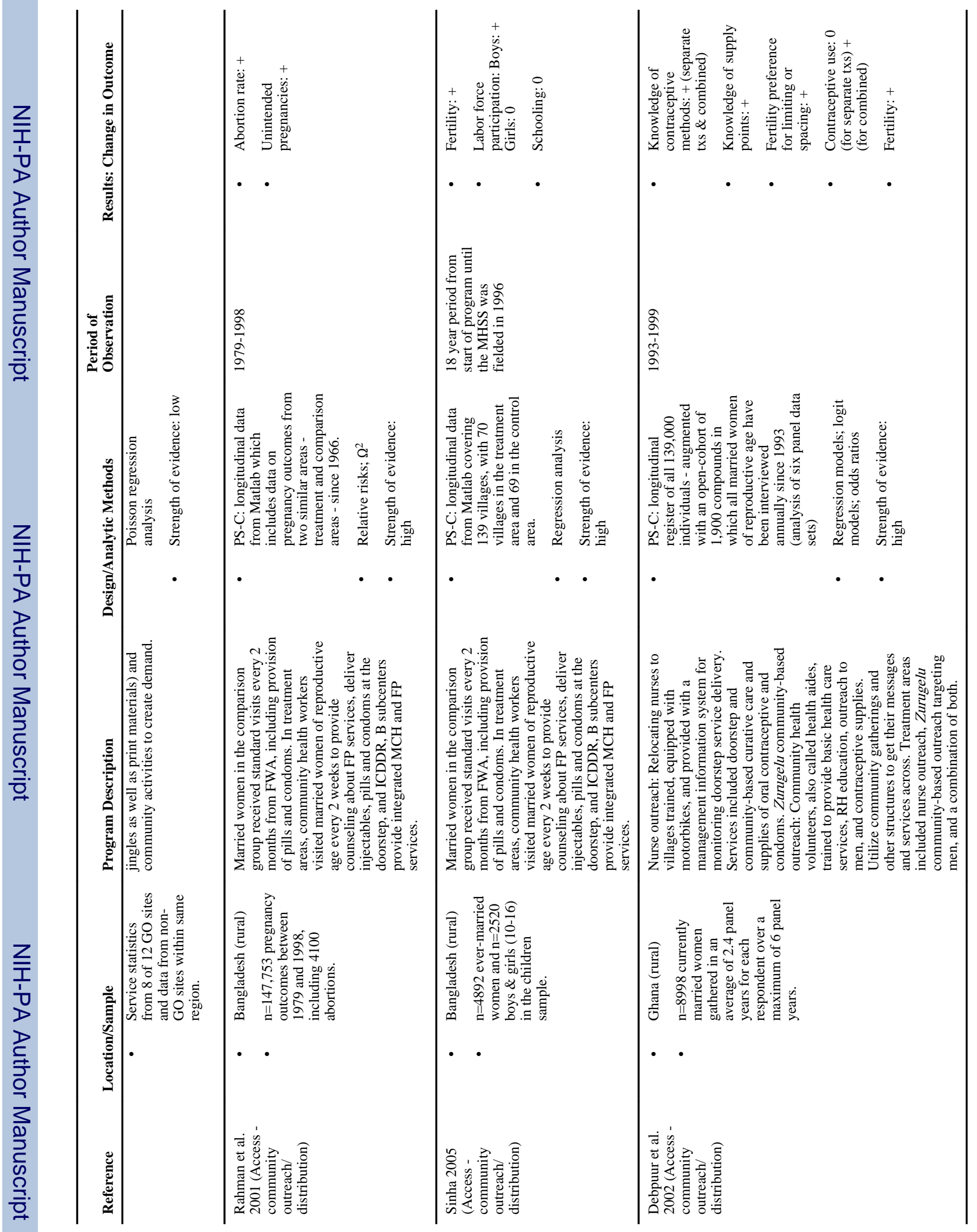




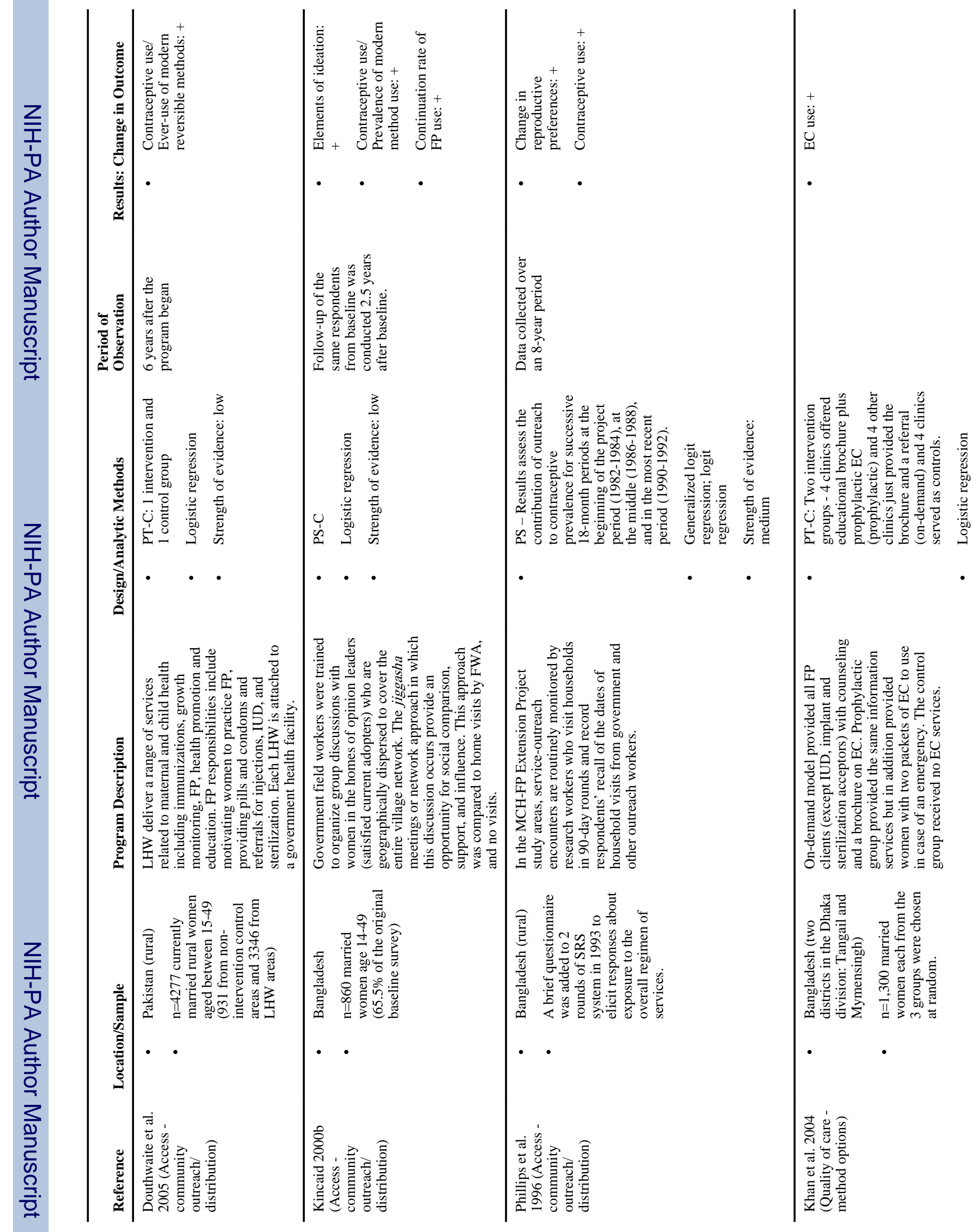




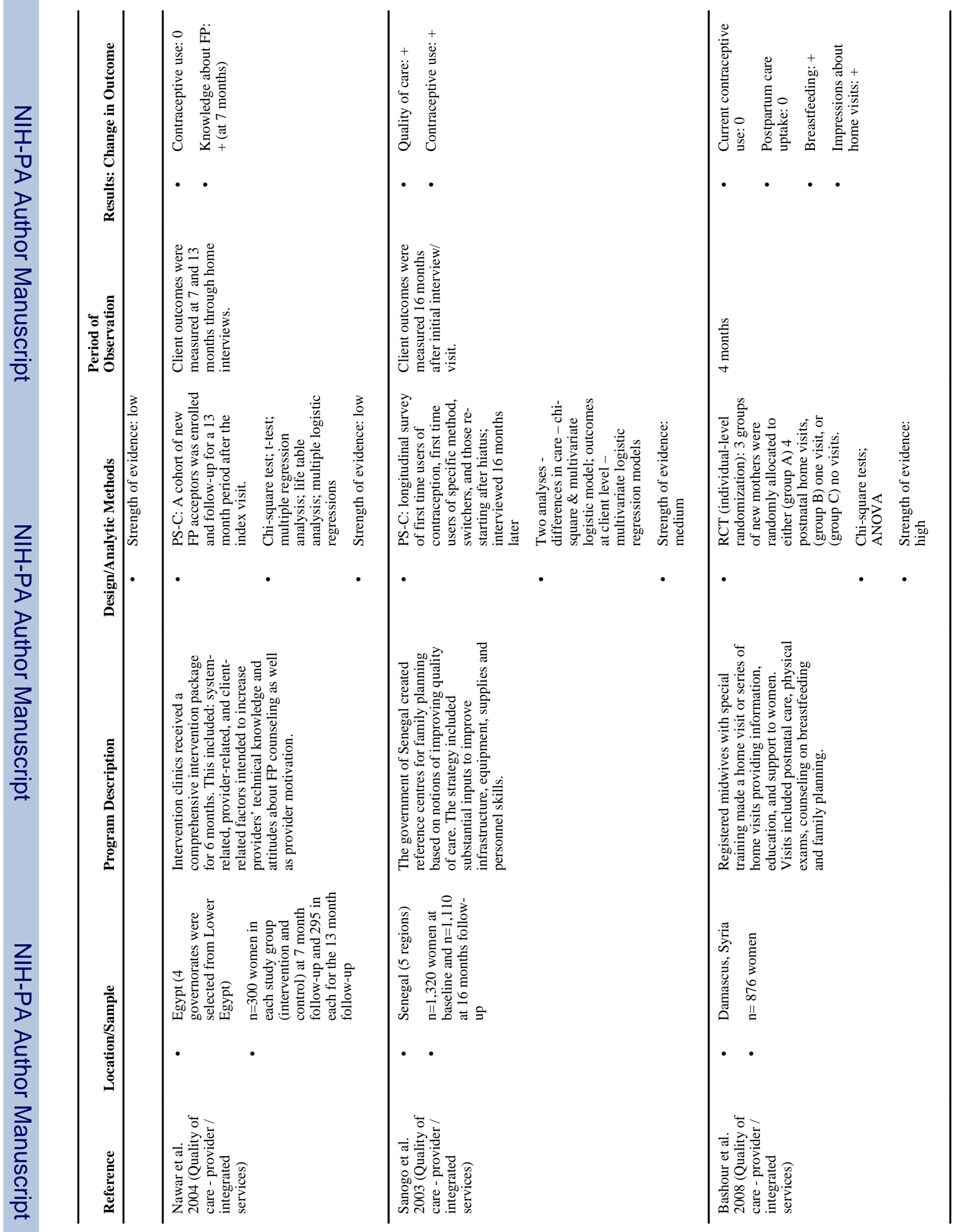




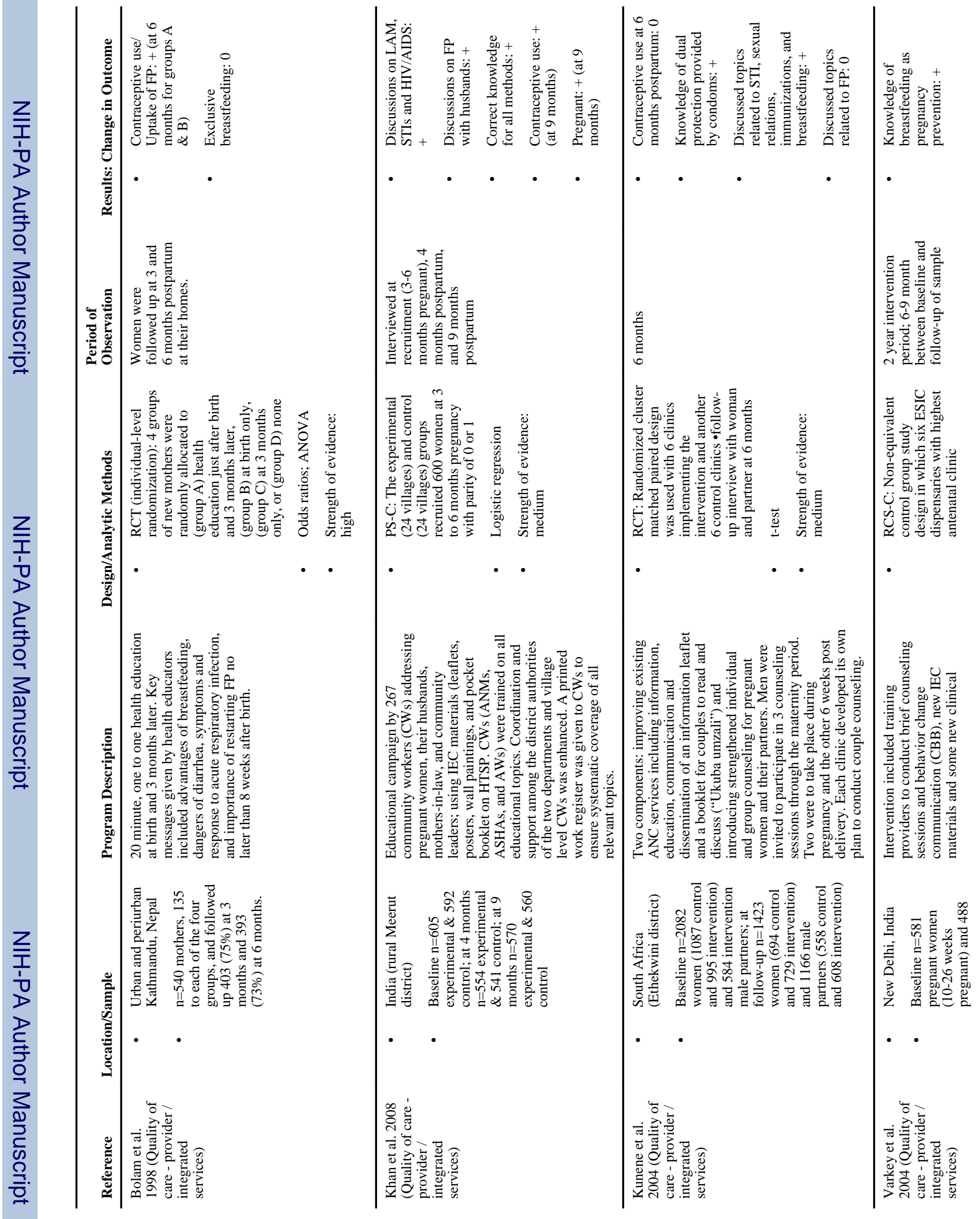




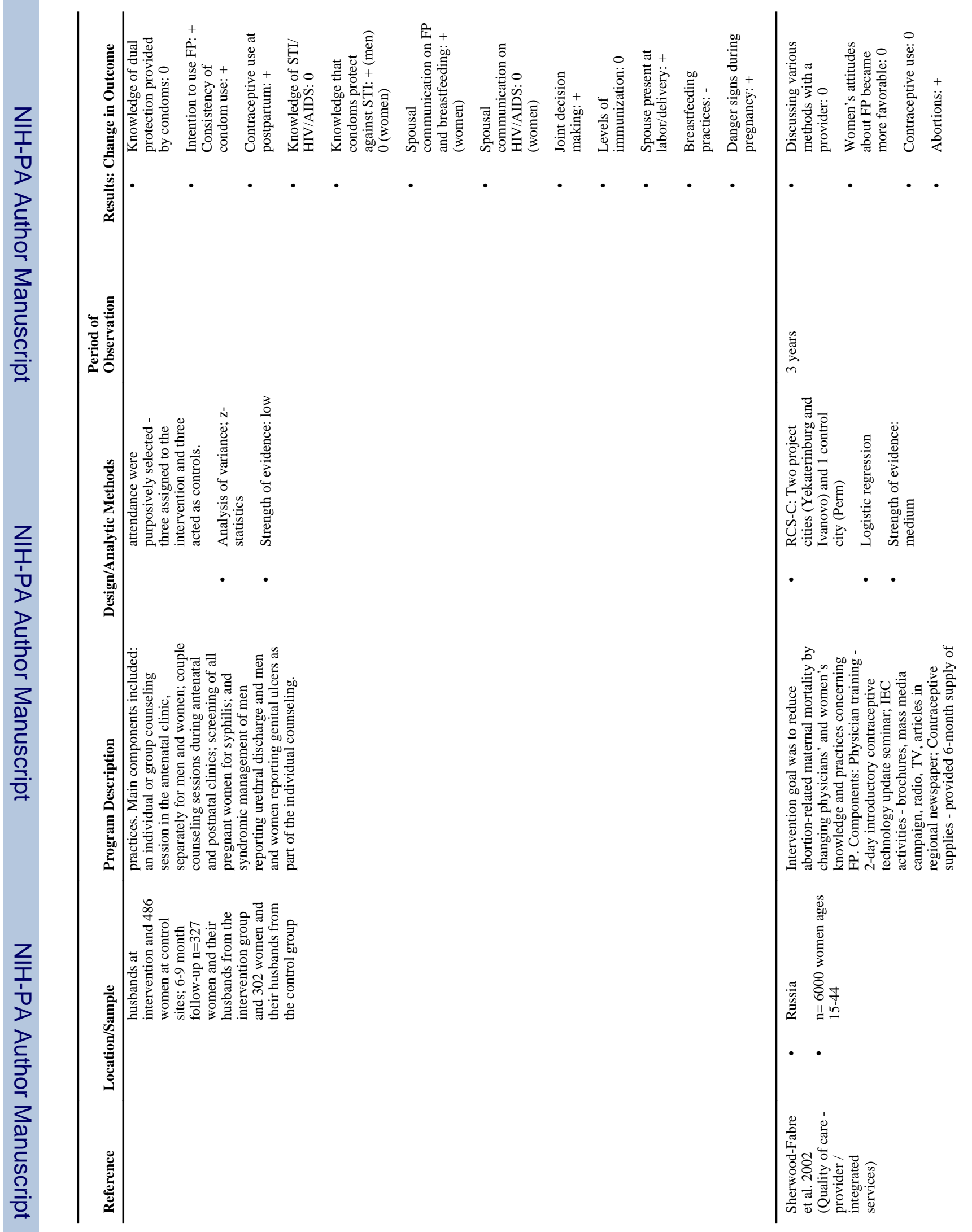


Mwaikambo et al.

Page 51

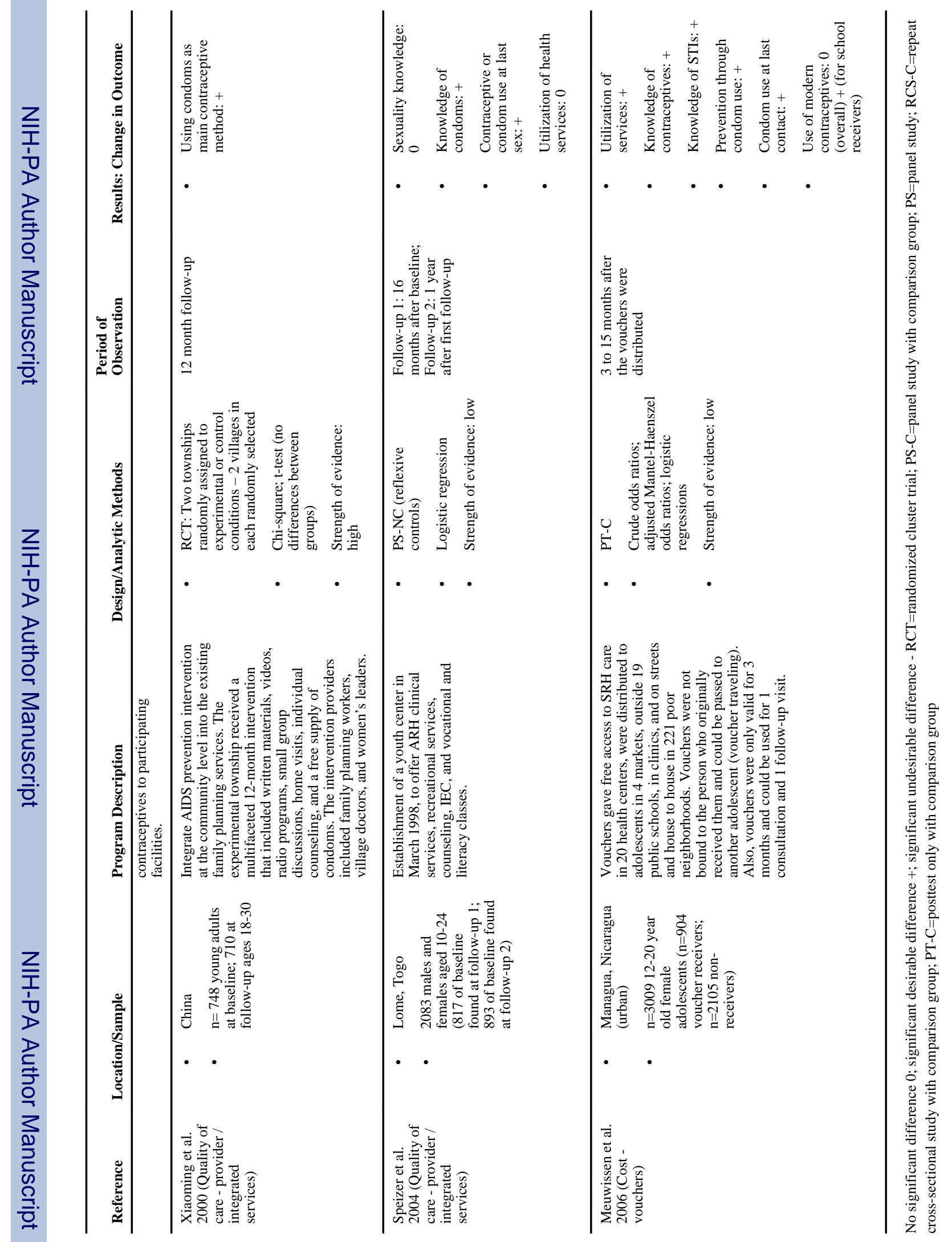

Stud Fam Plann. Author manuscript; available in PMC 2013 September 03. 